\title{
Effect of Concentric Annular Gap Flow on Wall Shear Stress of Stationary Cylinder Pipe Vehicle under Different Reynolds Numbers
}

\author{
Xiaomeng Jia, ${ }^{1}$ Xihuan Sun $\mathbb{D}^{1,2}$ and Jiaorong Song ${ }^{1}$ \\ ${ }^{1}$ Country. College of Water Resource Science and Engineering, Taiyuan University of Technology, Taiyuan, China \\ ${ }^{2}$ Jin Zhong University, Jinzhong, China \\ Correspondence should be addressed to Xihuan Sun; sunxihuan@tyut.edu.cn
}

Received 6 February 2020; Revised 13 April 2020; Accepted 28 April 2020; Published 15 May 2020

Academic Editor: Michalis Xenos

Copyright ( $) 2020$ Xiaomeng Jia et al. This is an open access article distributed under the Creative Commons Attribution License, which permits unrestricted use, distribution, and reproduction in any medium, provided the original work is properly cited.

The tube-contained raw material pipeline hydraulic transportation technology is an optimization and improvement of traditional hydraulic capsule pipeline (HCP) transport. It has the advantages of lower resource consumption, environmental protection, and less demand for human resources and has the ability to directly transport solids, liquids, and gases. The cylinder pipe vehicle is the core component of tube-contained raw material pipeline hydraulic transportation; its motion characteristics and energy consumption are affected by wall shear stress. When the cylinder pipe vehicle is stationary, the annular gap flow will affect the wall shear stress. This paper studies the wall shear stress and annular flow field distribution of a stationary cylinder pipe vehicle under different Reynolds numbers. The results show that as the Reynolds number increases, both the wall shear stress and the annular gap flow velocity show a gradually increasing trend. The wall shear stress and the velocity of the annular gap flow show some correlation, but there are differences in the trend of axial and circumferential wall shear stress along the length of the cylinder pipe vehicle. The research in this article will further improve the theoretical system of hydraulic conveyance of barrel-loading pipelines and provide a theoretical basis for the realization of industrial applications as soon as possible.

\section{Introduction}

Hydraulic capsule pipeline (HCP) transport technology was first proposed more than 60 years ago by the Alberta Research Center of Canada [1-7]. As a new mode of transportation, the hydraulic capsule pipeline transport technology mainly stores materials in capsules and transports them to their destinations through a pipeline. Compared with traditional transportation methods, HCP does not require the preparation and dehydration of the slurry (like slurry pipeline transportation) nor does it need to compress and decompose the moldings (like hydraulic transport of molding pipelines). Because the pipe is laid underground, it is concealed and not easily affected by weather conditions. The transport medium is water and it does not need to consume many fossil fuels. Compared with traditional transportation methods such as the railway and roads, it has the advantages of small investment, less environmental pollution, and less manpower required. Therefore, strengthening the research on HCP has important practical significance for changing traditional transportation methods in the future.

After more than half a century of research and development, HCP has achieved many positive research results. Newton et al. [8] used numerical calculations to study the velocity and pressure drop of the capsule with a length-todiameter ratio varying from 1 to 20 , but the calculation is limited to laminar flow, so it has certain limitations. Liu et al. $[9,10]$ believed that the entrance and exit head losses are ignored in the traditional capsule pressure drop theory; this assumption is applicable to long capsules but is not suitable for short capsules. They improved the traditional pressure drop theory by introducing a kinetic-energy correction factor $\alpha$ and verified the accuracy of the formula through 
physical experiments. Tomita et al. $[11,12]$ regarded a single capsule as a point mass and is concentric with the pipeline and used a characteristic method to numerically calculate the movement characteristics of the capsule in a straight tube, verifying the accuracy of the numerical calculation through physical experiments and then applying the calculation method to the movement of capsule trains. Vlasak [13] conducted an experimental investigation of the transport of a capsule of anomalous shape in a horizontal straight pipe. The variables include the velocity of a single capsule, the correlation between the speed of the capsule train and the velocity of liquid transport, and the pressure gradient of the capsule train during transportation. He found that when the Reynolds number was lower than $5 \times 10^{4}$, the velocity of the rigid capsule trains was greater than that of semirigid capsule trains, and the semirigid capsule trains had about a $20 \%$ lower pressure gradient than trains consisting of identical solid cylindrical capsules. Latto and Chow [14] conducted an analytical study of the hydrodynamic transport of cylindrical capsules in a vertical pipeline. In this experiment, the capsule-to-pipe diameter ratio varied from 0.42 to 0.82 , the capsule length-to-diameter ratio varied from 4 to 14 , and the relative transport density ratio of the capsule varied in the range of 1.15 to 1.7. The results show that as the diameter ratio or length to diameter ratio increased, the velocity and pressure drop gradient of the capsule also showed a gradually increasing trend. As the density of the capsules increased, the velocity decreased but the pressure gradient increased. According to the flow mechanism, Ulusarslan et al. [15-17] analyzed the pressure gradient of the spherical capsule train in a horizontal circular pipe; a related experiment was subsequently conducted that used a spherical polyethylene capsule with a density equal to water to replace an ice capsule. It was found that the total pressure gradient can be divided into the pressure gradient caused by homogeneous flow and the pressure gradient caused by rotational kinetic energy. When the capsule transport concentration is low, the pressure loss caused by the rotational kinetic energy is small, while the pressure loss caused by the homogeneous flow is large. Robert and Nivedita [18] used the front tracking method to study deformable capsules at different Reynolds numbers. The results showed that the size of the capsule and the Reynolds number will affect the deformation, migration speed, and pressure loss of the capsules.

With the rapid development of computers, numerical simulation has been applied more often to HCP transport. Mohamed et al. [19] used three different turbulence models to study the turbulent flow characteristics around a single concentric long capsule in a straight pipe. The three models are Baldwin-Lomax, $k-\varepsilon$, and $k-\omega$; they were used to calculate the drag coefficient, the pressure gradient, and the velocity distribution in the annular gap between the capsule and the pipe, respectively. The simulation results are highly consistent with the experiment results. Asim [20] used a computational fluid dynamics (CFD) tool to numerically simulate the hydraulic characteristics of a single capsule with different diameter ratios. The simulation results show that as the diameter ratios increase, the pressure gradient between the upstream and downstream sections gradually increases, and the water flow velocity in the annular gap also increases. Then, it numerically simulated the velocity, static pressure, and vorticity distribution of a single capsule in a horizontal straight pipe, bent pipe, and vertical pipe section, and the pipeline system of the HCP transport was optimized based on the simulation results. Asim et al. [21, 22] extended their work to numerically simulate the hydraulic characteristics of different shaped capsules in a straight pipeline. The above research results provide theoretical guidance for the application of HCP transportation technology and lay the foundation for the future development of the capsule train.

Sun et al. proposed tube-contained raw material pipeline hydraulic transportation technology based on traditional hydraulic capsule pipeline transport. The tube-contained raw material pipeline hydraulic transportation technology is a new transportation method in which the material is placed in a closed container (generally a cylindrical pipe vehicle), while six support bodies are symmetrically installed at both ends of the container to make it move concentrically in the pipeline, which can reduce the collision and friction of the container against the pipe wall. Then, water pressure is used to transport the container to the destination. When the container is stationary in the pipeline, the water flow through the container belongs to the flow around a cylinder $[23,24]$. The container and the pipe wall form a concentric annular gap, and the flow of water through it belongs to the annular gap flow problem [25-27]. The container wall will form a boundary layer due to the effect of viscous force, which belongs to the static boundary layer problem [28, 29]. When the container moves in the pipeline, it also involves the problem of dynamic boundary and fluid-solid coupling $[30,31]$. The new transportation technology involves automation, transportation, and other issues. Therefore, it is of great significance to strengthen the research on tube-contained raw material pipeline hydraulic transportation technology.

Sun et al. [32] first built a cylinder pipe vehicle transport system consisting of four parts: a launching device, a power unit, a transport pipeline, and a recovery device. The system was used to test the start-up conditions of the cylinder pipe vehicle and analyze the relevant factors affecting their movement. On the basis of Sun's experiment, $\mathrm{Li}$ et al. [33-35] studied the hydraulic characteristics of the cylinder pipe vehicle with different loads and models and obtained the velocity of the cylinder pipe and the pressure loss along the pipeline and calculated the energy loss of the entire system. As described by Wang et al. [36], the motion of the cylinder pipe can be classified into three stages. They established related mathematical models to analyze the relationship between the speed of the cylinder pipe vehicle, the velocity of the annulus gap flow, and the velocity of the water in the pipeline, and the accuracy of the mathematical model was verified by physical experiments. Zhang et al. [37, 38] carried out a numerical simulation on the cylinder pipe vehicle moving in the straight pipe section. It was found that the axial flow velocity of the upstream and downstream sections is larger at the inner wall of the pipe, the flow velocity near the center of the pipe is smaller, and the radial 
flow rate tends to first increase and then decrease from the inner wall to the center of the pipe, while the circumferential velocity is symmetrically distributed near the support of the cylinder pipe vehicle.

Previous research on cylinder pipe vehicles has mostly focused on flow velocity and pressure gradients [39, 40]. However, there are relatively few studies on the wall shear stress of the cylinder pipe vehicle. A concentric annular gap is formed between the cylinder pipe vehicle and the pipe wall. When the water flows into the annular gap, the cylinder pipe vehicle is subjected to the wall shear stress acting on it. The existence of wall shear stress has an impact on the motion and energy loss of the cylinder pipe vehicle. Therefore, strengthening the analysis and research on the wall shear stress of the cylindrical pipe vehicle can provide a certain theoretical basis for reducing the transportation energy consumption and can further enrich and improve the theoretical system of tube-contained raw material pipeline hydraulic transportation.

The main content of the article is as follows. Section 2 is the theoretical analysis, which mainly includes the introduction of the cylinder pipe vehicle structure and the force analysis. Section 3 introduces the experimental system and procedures. Section 4 analyzes and discusses the experimental results. The main conclusions are given in Section 5.

\section{Theoretical Analysis}

2.1. Structure of the Cylinder Pipe Vehicle. As the core component of tube-contained raw material pipeline hydraulic transportation, the cylinder pipe vehicle is mainly composed of a container, two sealing covers, and support bodies. The container is made of cylindrical plexiglass, which is used to hold the material, avoiding contact between the material and the conveying liquid. In order to make the cylinder pipe vehicle move concentrically in the pipeline, support bodies are installed on the sealing covers. By adding support bodies, the friction between the cylindrical pipe vehicle and the pipeline is reduced, thereby extending the service life of the pipeline. The entity structure of the cylinder pipe vehicle is shown in Figure 1.

The model of the cylinder pipe vehicle used in this experiment is $150 \mathrm{~mm} \times 80 \mathrm{~mm}$, where 150 indicates the length of the cylinder pipe vehicle $(L)$, and 80 is the diameter of the cylinder pipe vehicle $(D)$. Each sealing cover is installed with three support bodies, and the support bodies are distributed at an angle of $120^{\circ}$.

2.2. Force Analysis of the Cylinder Pipe Vehicle. Since the cylinder pipe vehicle is concentric with the pipeline, the force acting on the cylinder pipe vehicle can be divided into two directions, namely, vertical and parallel to the pipe axis. The forces that are vertical to the axis are self and material gravity $G$, buoyancy force $F_{c}$, and support force $F_{n}$ of the pipeline to the cylinder pipe vehicle. The vertical force does not directly affect the movement of the cylinder pipe vehicle. The forces parallel to the axis are the pressure $F_{p 1}, F_{p 2}$ on the front and rear seal cover, the wall shear force $F_{\tau}$, and the friction force $F_{f}$ between the support bodies and the pipe wall. The force parallel to the axis direction has a direct effect on the movement of the cylinder pipe vehicle. The specific force is shown in Figure 2.

(1) Gravity $G$ includes the weight of the cylinder pipe vehicle and internal materials, which can be expressed as follows:

$$
G=\left(m_{c}+m_{m}\right) g,
$$

where $m_{c}$ is the weight of the cylinder pipe vehicle and $m_{m}$ is the weight of the material.

(2) Buoyancy force $F_{c}$ is opposite to the direction of gravity $G$, which is vertical to the pipeline axis, which can be expressed as follows:

$$
F_{c}=\rho g V_{c} \text {, }
$$

where $\rho$ is the density of the liquid and $V_{c}$ is the volume of the cylinder pipe vehicle.

(3) The force on the end face of the cylinder pipe vehicle is shown in Figure 3. When gravity $G$ is equal to buoyancy force $F_{c}$, the cylinder pipe vehicle is equivalent to being suspended in the pipeline, and it can be considered that the support force is zero. When $G \geqq F_{c}$, the cylinder pipe vehicle sinks, and the force on the bottom support is much larger than the force on the upper support, namely, $F_{n 2}=F_{n 3} \gg F_{n 1}$, and $F_{n 1}$ can be ignored. The support force is $4 F_{n 2}$, which can be expressed as follows:

$$
F_{n}=G-F_{c}=4 F_{n 2} \cos \theta \text {, }
$$

where $\theta$ represents the angle between the support force $F_{n 2}$ and $F_{c}$.

When $G \leqq F_{c}$, the cylinder pipe vehicle floats up and the force on the upper support is much larger than the force on the bottom support, namely, $F_{n 2}=F_{n 3} \ll F_{n 1}$; $F_{n 2}$ and $F_{n 3}$ can thus be ignored. The support force is $2 F_{n 1}$, which can be expressed as follows:

$$
F_{n}=F_{c}-G=2 F_{n 1} \text {. }
$$

(4) The main driving force of the cylindrical pipe vehicle comes from the pressure difference between the front and rear end faces. Generally, the pressure difference force is expressed by the following formula:

$$
F_{\Delta p}=F_{p 1}-F_{p 2} \text {, }
$$

where $F_{p 1}$ and $F_{p 2}$ represent the pressure of the water flow on the front and rear end faces, respectively (the direction of the water flow is the rear end face of the cylinder pipe vehicle).

If $F_{p 1}-F_{p 2}=\Delta P \cdot A$, then equation (5) can be expressed as follows:

$$
F_{\Delta P}=\Delta P \cdot A=\frac{Q^{2} \rho g}{\mu_{c}^{2} A},
$$




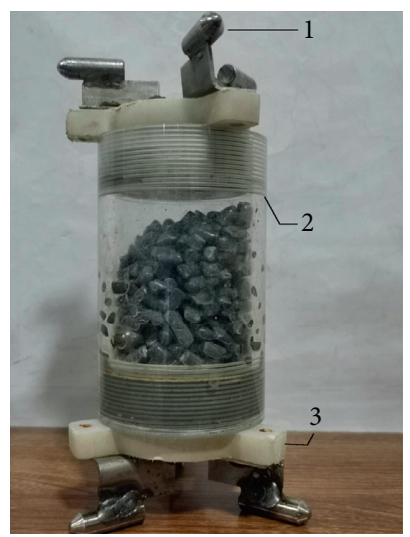

(a)

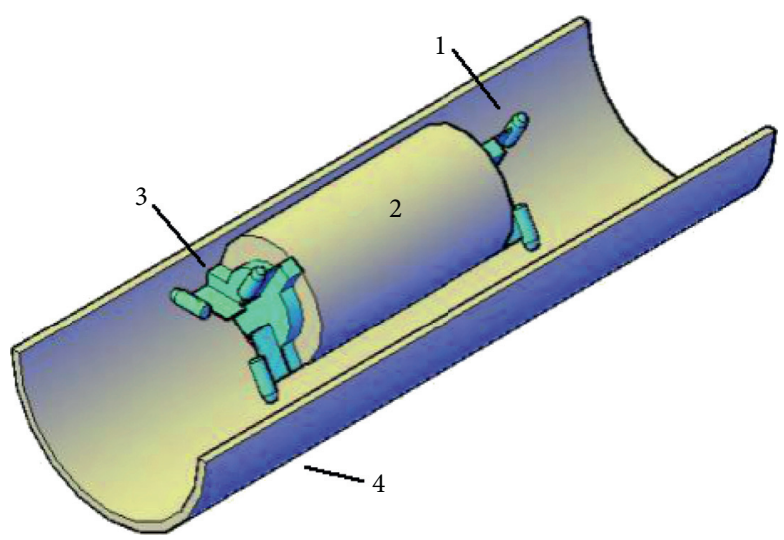

(b)

Figure 1: A structural sketch of the cylinder pipe vehicle. (a) Physical model. (b) Geometric model. Note: 1, support body; 2, container; 3, sealing cover; 4 , pipeline.

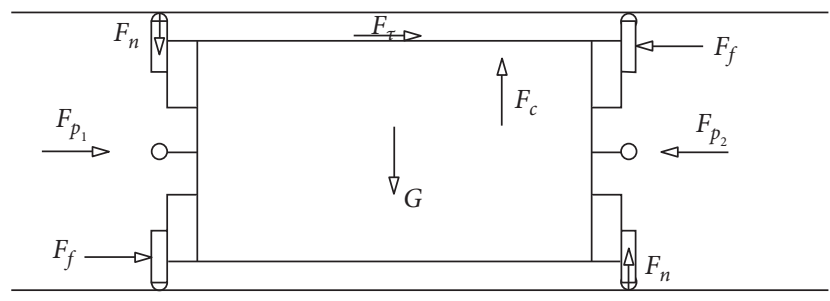

FIGURE 2: Schematic diagram of force of the cylinder pipe vehicle.

where $\Delta P$ represents the pressure difference between the front and rear end faces, $A$ is the end face area, $Q$ is the flow rate in the pipeline, and $\mu_{c}$ is the flow coefficient in the pipeline.

(5) From the previous analysis, when the buoyancy is less than the weight of the cylinder pipe vehicle, $F_{n 1}=0$, so the friction on the upper support can be regarded as zero. The friction force can be expressed as follows:

$$
F_{f}=\mu F_{n 2}=\mu \frac{G-F_{c}}{4 \cos \theta},
$$

where $\mu$ represents the friction factor between the support bodies and the pipe wall. When the buoyancy is larger than the weight of the cylinder pipe vehicle, $F_{n 2}=F_{n 3}=0$, so the friction on the bottom support can be regarded as zero. The friction force can be expressed as follows:

$$
F_{\tau}=\frac{\lambda_{c}}{8} \rho\left(v_{a}-v_{c}\right)^{2}
$$

(6) If the flow velocity of the annular gap is greater than the running speed of the cylinder pipe vehicle, the wall shear force of the cylinder pipe vehicle is the driving force. When the flow velocity of the annular gap is less than the running speed of the cylinder pipe vehicle, the wall shear force of the cylinder pipe

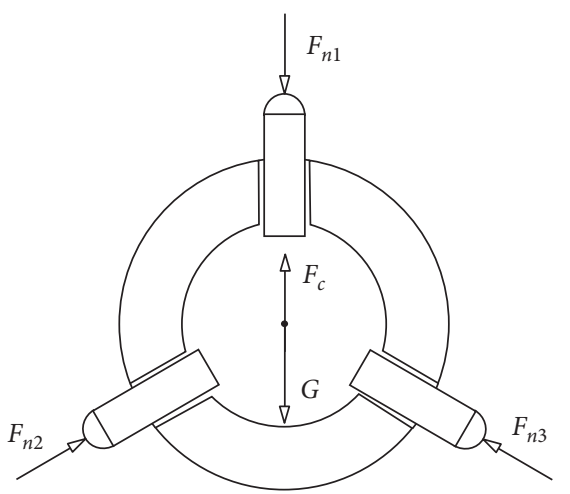

Figure 3: The force diagram of each end face.

vehicle is the resistance. The wall shear force can be expressed as follows:

$$
F_{\tau}=\frac{\lambda_{c}}{8} \rho\left(v_{a}-v_{c}\right)^{2}
$$

where $\lambda_{c}$ represents the flow resistance coefficient, which is related to the material of the inner wall of the pipe and the physical properties of the fluid; $v_{a}$ represents the velocity of the annular gap flow; and $v_{c}$ represents the velocity of water in the pipe.

2.3. Analysis of the Concentric Annular Gap Flow Velocity. Annular gap flow can be divided into two types according to the existence of relative motion of the solid side wall: one is the static boundary gap flow and the other is the dynamic boundary gap flow. The research in this paper considers static boundary gap flow. The mathematical model of the cylinder pipe vehicle is shown in Figure 4.

Assuming the cylinder pipe vehicle is infinitely long, the water flow in the pipe can be considered as a steady uniform flow. Establishing a coordinate system as shown in Figure 4, we obtain the governing equation (10) of annular gap flow at static boundary according to the Navier-Stokes equation: 


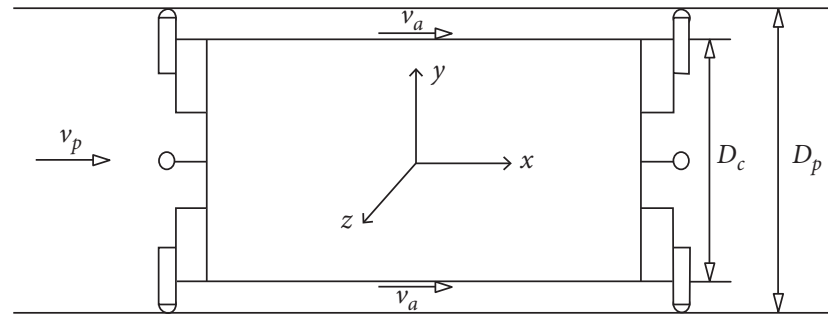

Figure 4: A schematic diagram of the mathematical model.

$$
\begin{aligned}
\frac{\partial u_{x}}{\partial t} & +u_{x} \frac{\partial u_{x}}{\partial x}+u_{y} \frac{\partial u_{x}}{\partial y}+u_{z} \frac{\partial u_{x}}{\partial z} \\
& =f_{x}-\frac{1}{\rho} \frac{\partial P}{\partial x}+v\left(\frac{\partial^{2} u_{x}}{\partial x^{2}}+\frac{\partial^{2} u_{x}}{\partial y^{2}}+\frac{\partial^{2} u_{x}}{\partial z^{2}}\right),
\end{aligned}
$$

where $f_{x}$ is the mass force; $P$ is the dynamic water pressure; $u_{x}$, $u_{y}$, and $u_{z}$ are the partial velocities along the $X$-axis, $Y$-axis, and $Z$-axis, respectively; and $v$ is the kinematic viscosity coefficient.

Because the water flow in the annular gap is constant, only the direction of movement along the axis of the pipeline is considered.

$$
\begin{gathered}
\frac{\partial u_{x}}{\partial t}=0, \\
u_{y}=u_{z}=0, \\
\frac{\partial u_{y}}{\partial y}=\frac{\partial u_{z}}{\partial z}=0, \\
u_{y} \frac{\partial u_{x}}{\partial y}=u_{z} \frac{\partial u_{x}}{\partial z}=0 .
\end{gathered}
$$

The only force of mass on water is gravity:

$$
f_{x}=0 \text {. }
$$

According to the continuity equation (13):

$$
\frac{\partial u_{x}}{\partial x}+\frac{\partial u_{y}}{\partial y}+\frac{\partial u_{z}}{\partial z}=0
$$

Substituting equations (11)-(13) into equation (10), equation (10) can be expressed as follows:

$$
\frac{1}{\rho} \frac{\partial P}{\partial x}=v\left(\frac{\partial^{2} u_{x}}{\partial y^{2}}+\frac{\partial^{2} u_{x}}{\partial z^{2}}\right)
$$

Because concentric annular gap flow is axisymmetric, both the $Y$-axis and $Z$-axis can be regarded as along the radial direction. Equation (14) can be expressed as

$$
\frac{\partial^{2} u_{x}}{\partial r^{2}}=-2 \frac{\Delta P}{L \rho v} .
$$

Integrating equation (15), we get

$$
u_{x}=-\frac{\Delta P}{4 L \rho v} r^{2}+c_{1} r+c_{2} .
$$

According to boundary conditions, we can get the following equation:

$$
u_{x}=-\frac{\Delta P}{4 L \rho v}\left(r-r_{p}\right)\left(r-r_{c}\right)
$$

We can calculate the flow of the annular gap according to equation (17):

$$
Q=\int_{r_{c}}^{r_{p}} 2 \pi u_{x}\left(r-r_{c}\right) \mathrm{d} r=-\frac{\pi \Delta p}{24 L v \rho} K,
$$

where $K=r_{p}^{4}+8 r_{p}^{3} r_{c}-12 r_{p}^{2} r_{c}^{2}-8 r_{p} r_{c}^{3}-r_{c}^{4}, r_{p}$ is the radius of the pipeline, and $r_{c}$ is the radius of the cylinder pipe vehicle.

\section{Experimental System and Procedures}

The experimental system consists of four parts: an experimental pipeline, regulating equipment, a power system, and measuring equipment. The experimental system is shown in Figure 5.

The experimental pipeline is composed of plexiglass with an inner diameter of $100 \mathrm{~mm}$ and a wall thickness of $5 \mathrm{~mm}$. In order to reduce the refraction of the pipeline to the laser during the experiment, a rectangular water jacket is added in the test pipe section. The regulating equipment includes an electromagnetic flowmeter and a gate valve. The power required for the experiment is provided by the centrifugal pump. The measuring equipment consists of the laser Doppler anemometry and the cylinder pipe vehicle force measuring device, as shown in Figure 6.

In order to measure the wall shear stress, a cross section is set up $30 \mathrm{~mm}$ along the direction of the cylinder pipe vehicle, as shown in Figure 7(a). A measurement point is arranged clockwise every $30^{\circ}$ along the circumference of the cross section, and the strain flower is pasted on each measurement point, as shown in Figure 7(b). The coordinates of each measurement point are shown in Table 1.

An annular gap is formed between the wall surface of the cylinder pipe vehicle and the wall surface of the pipeline; the width of the gap is $L=10 \mathrm{~mm}$. In order to measure the annular gap flow field, five measuring rings were arranged along the radius of the cross section, as shown in Figure 8 . The pipe radius intersects the measuring ring every $30^{\circ}$ clockwise, the intersection point is the measuring point, and a total of 60 measuring points are arranged for each cross section.

This experiment studies the change in wall shear stress of a cylinder pipe vehicle under different Reynolds numbers. The selected Reynolds numbers $(\mathrm{Re})$ are $\operatorname{Re}_{1}=115,513$, $\operatorname{Re}_{2}=154,018, \operatorname{Re}_{3}=192,522$, and $\operatorname{Re}_{4}=231,027$. The Reynolds number can be calculated according to equation (19), and the flow rate corresponding to the experimental Reynolds number is $30 \mathrm{~m}^{3} / \mathrm{h}, 40 \mathrm{~m}^{3} / \mathrm{h}, 50 \mathrm{~m}^{3} / \mathrm{h}$, or $60 \mathrm{~m}^{3} / \mathrm{h}$.

$$
\operatorname{Re}=\frac{\rho v d}{\eta}=\frac{v d}{v}=\frac{4 Q}{\pi D v},
$$

where $p$ is the liquid density, $v$ is the velocity of water in the pipe, $d$ is the pipe radius, $\eta$ is the dynamic viscosity, $v$ is the kinematic viscosity, $Q$ is the flow rate in the pipe, and $D$ is the pipe diameter.

The specific experimental plan is shown in Table 2 .

The strain flower is glued to the measuring point and connected to the force measuring system inside the cylinder pipe vehicle. Meanwhile, a waterproof coating is applied on the surface of the strain flower. Fix the cylinder pipe vehicle to the test tube to 


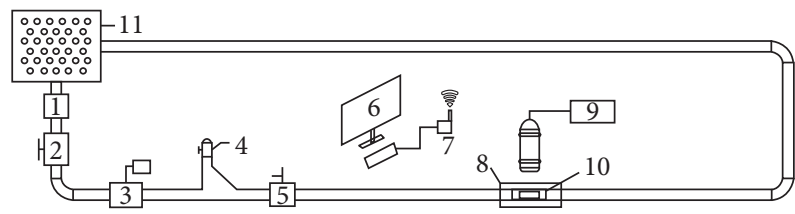

Figure 5: Experimental system. Note: 1, centrifugal pump; 2, regulating valve; 3, electromagnetic flowmeter; 4, the feeding device; 5, brake device; 6 , computer; 7 , measuring force receiving device; 8 , rectangular water jacket; 9 , laser Doppler anemometry; 10 , the cylinder pipe vehicle; 11 , water tank.

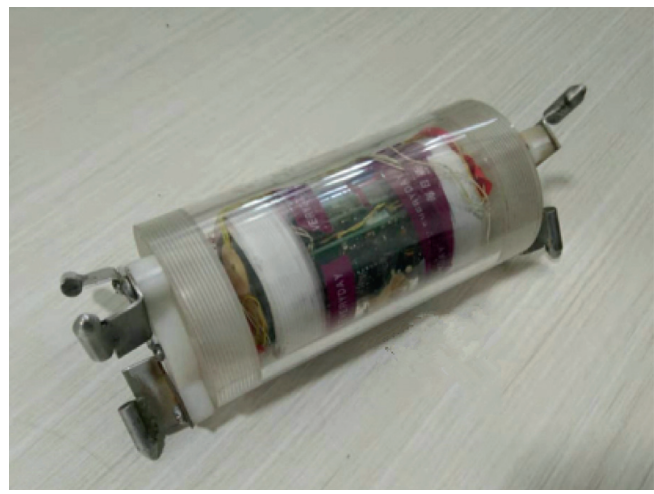

(a)

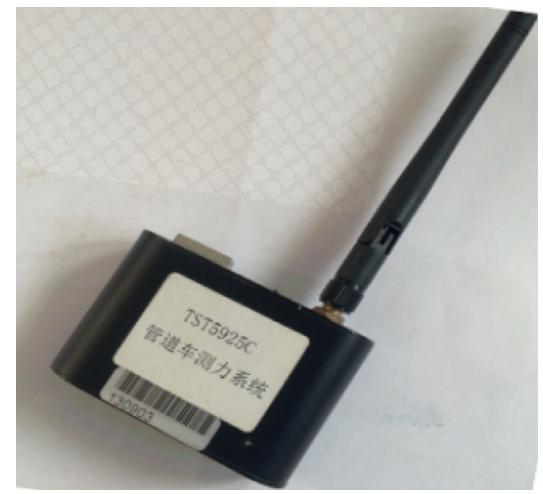

(b)

FIGURE 6: The cylinder pipe vehicle force measurement equipment. (a) Force measuring device. (b) Signal receiving equipment.

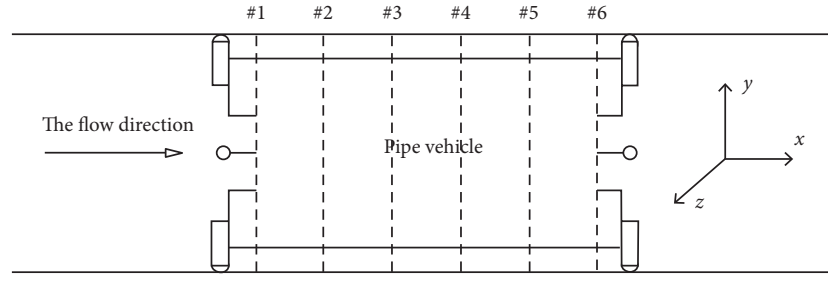

(a)

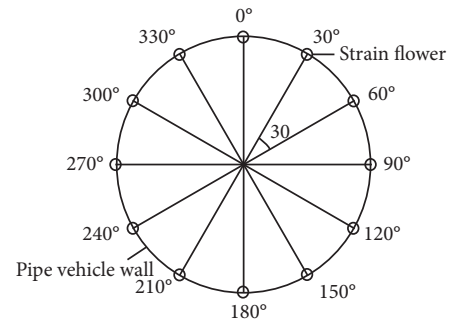

(b)

Figure 7: A cross section layout and measurement point distribution. (a) A cross section layout. (b) Measurement point distribution.

TABLE 1: Coordinates of measuring points.

\begin{tabular}{|c|c|c|c|c|c|c|c|c|}
\hline Number of measuring points & $r(\mathrm{~mm})$ & $\Phi\left(^{\circ}\right)$ & Number of measuring points & $r(\mathrm{~mm})$ & $\Phi\left(^{\circ}\right)$ & Number of measuring points & $r(\mathrm{~mm})$ & $\Phi\left({ }^{\circ}\right)$ \\
\hline 1 & 40 & 0 & 5 & 40 & 120 & 9 & 40 & 240 \\
\hline 2 & 40 & 30 & 6 & 40 & 150 & 10 & 40 & 270 \\
\hline 3 & 40 & 60 & 7 & 40 & 180 & 11 & 40 & 300 \\
\hline 4 & 40 & 90 & 8 & 40 & 210 & 12 & 40 & 330 \\
\hline
\end{tabular}

Note. $r$ represents the radius; $\Phi$ represents the angle on the cross section.

form a complete circuit. Fill the water tank and add tracer particles, then start the centrifugal pump to inject water from the water tank into the pipeline, and adjust the Reynolds number by adjusting the device. After the water flow in the pipeline is stable, the annular gap flow field and the wall shear stress are measured.

\section{Results and Discussion}

4.1. Distribution of Concentric Annular Gap Flow. The magnitude and distribution of the wall shear stress are affected by the annular gap flow. Therefore, first we must analyze the change in annular gap flow under different Reynolds numbers. The calculated values and experimental values of the average velocity of the annular gap flow under different Reynolds numbers are shown in Table 3.

It can be seen from Table 3 that the experimental values are in agreement with the calculated values, and the maximum relative error between the experimental values and calculated values does not exceed $4.63 \%$. As the Reynolds number increases, the average velocity of the annular gap 


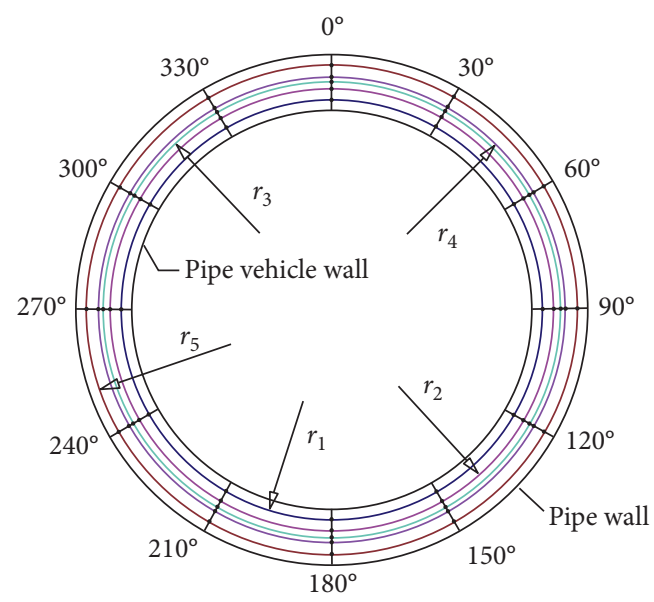

FIGURE 8: Velocity measuring point arrangement. Note: $r_{1}=d_{c}+1 / 5 L=42 \mathrm{~mm}, r_{2}=d_{c}+2 / 5 L=44 \mathrm{~mm}, r_{3}=d_{c}+1 / 2 L=45 \mathrm{~mm}, r_{4}=d_{c}+3 /$ $5 \mathrm{~L}=42 \mathrm{~mm}$, and $r_{5}=d_{c}+4 / 5 \mathrm{~L}=48 \mathrm{~mm} ; d_{c}$ represents the radius of the cylinder pipe vehicle.

TABLe 2: Experimental plan.

\begin{tabular}{|c|c|c|c|}
\hline Piped vehicle & Reynolds number & Corresponding flow $\left(\mathrm{m}^{3} / \mathrm{h}\right)$ & Status \\
\hline & 115513 & 30 & \multirow{4}{*}{ Stationary } \\
\hline Length: 150 (mm) & 154018 & 40 & \\
\hline Radius: 80 (mm) & 192522 & 50 & \\
\hline & 231027 & 60 & \\
\hline
\end{tabular}

flow also gradually increases. This is mainly because as the Reynolds number increases, the water flow velocity in the pipeline increases. According to the continuity equation $A_{1} V_{1}=A_{2} V_{2}$, when the cross-sectional area remains unchanged, the annular gap flow velocity has a positive correlation with the water flow velocity in the pipeline-with the increase in the water flow velocity in the pipeline, the annular gap flow velocity value also increases.

Water flow in the annular gap can be decomposed into three directions, namely, the axial velocity along the pipe axis, the radial velocity along the pipe diameter direction, and the circumferential velocity along the tangent to the circumferential section.

4.1.1. Axial Velocity Distribution. The axial velocity is positive along the direction of the water flow; otherwise, it is negative. Take the Reynolds numbers $\operatorname{Re}_{1}=115,513$ and $\mathrm{Re}_{3}=192,522$ as an example to analyze the axial velocity distribution. The contour map of the axial velocity of each section is shown in Figure 9.

By comparing the contour map of the axial velocity of the annular gap under different Reynolds numbers, we see that with increases in the Reynolds number in the pipe, the axial velocity value also gradually increases. The axial flow velocity changes most drastically at Sections \#1 and \#3. This is mainly because Section \#1 is located at the entrance of the annular gap. When the water flow enters the annular gap, the cross section suddenly shrinks, the water flow is rapidly compressed, and the flow rate changes drastically. Moreover, Section \#1 is closer to the support body, and a vortex is generated after the water flows
TABLE 3: Experimental values and calculated values of average velocity of annular gap flow.

\begin{tabular}{lcc}
\hline $\mathrm{Re}$ & Experimental values $(\mathrm{m} / \mathrm{s})$ & Calculated values $(\mathrm{m} / \mathrm{s})$ \\
\hline $\mathrm{Re}_{1}=115,513$ & 2.81 & 2.94 \\
$\mathrm{Re}_{2}=154,018$ & 3.75 & 3.93 \\
$\mathrm{Re}_{3}=192,522$ & 4.71 & 4.91 \\
$\mathrm{Re}_{4}=231,027$ & 5.62 & 5.87 \\
\hline
\end{tabular}

through the support body. The vortex spreads to Section \#1, which makes the axial flow velocity at this section change more drastically. Section \#6 is located at the exit of the annular gap. When the water flows out of the annular gap, the cross section suddenly expands and the water flow diffuses into the pipe. The flow pattern also changed drastically. Some of the water flowing out of the annular gap will be returned to Section \#6 after being blocked by the support body, which makes the flow velocity change at Section \#6 more complicated. So, the axial flow velocity changes more drastically in this section. After the water flows into the annular gap, the water flow is redistributed by the restriction of the pipe wall and the pipe vehicle wall, and the flow state gradually stabilizes. Therefore, the axial velocity fluctuations at Sections \#2-5 are relatively small. It can also be seen from Figure 9 that as the Reynolds number increases, the velocity gradient near the pipe vehicle wall also gradually increases. The instability of the annular gap flow increases with the increase in the Reynolds number, that is, the fluctuation in the flow of each cross section increases [41-43]. The highvelocity region of water flow near the pipe vehicle wall is larger than that near the pipe wall. This is because the water flow near the pipe wall is mainly affected by the viscous resistance, and 


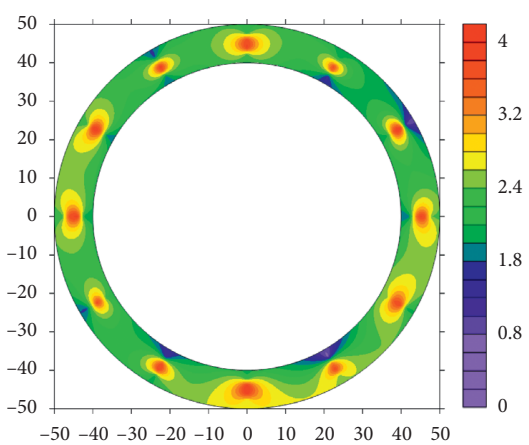

$\left(a_{1}\right)$ Section \#1

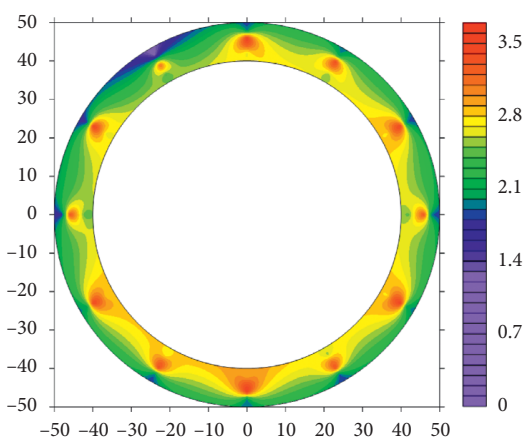

$\left(\mathrm{a}_{4}\right)$ Section \#4

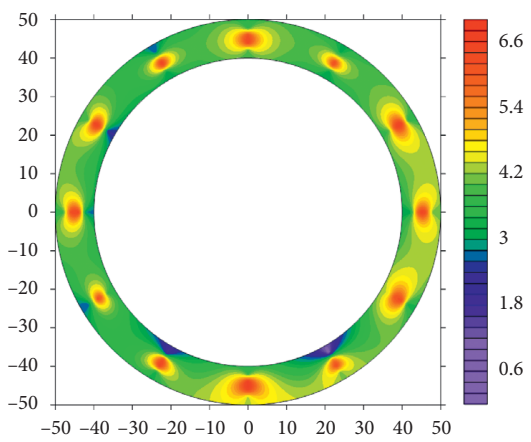

$\left(b_{1}\right)$ Section \# 1

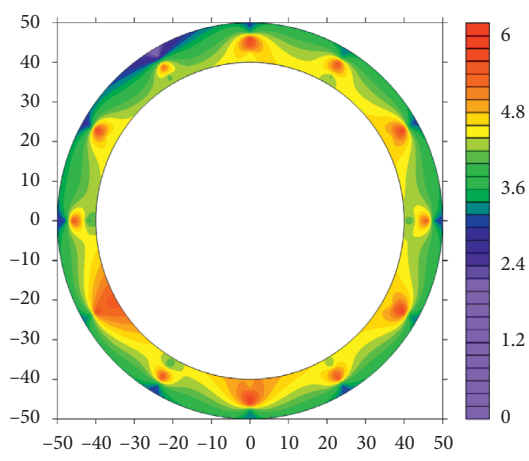

$\left(\mathrm{b}_{4}\right)$ Section \#4

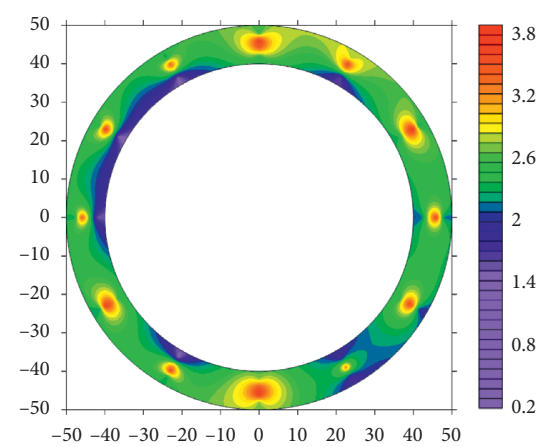

$\left(\mathrm{a}_{2}\right)$ Section \#2

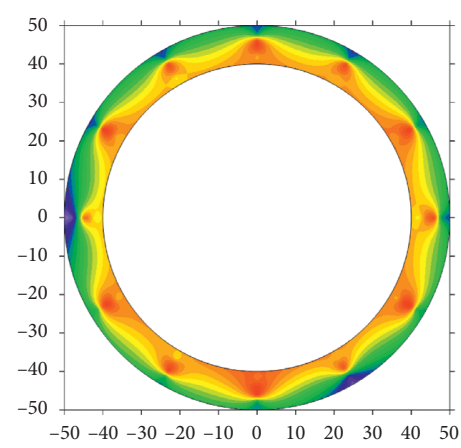

$\left(a_{5}\right)$ Section \#5

(a)

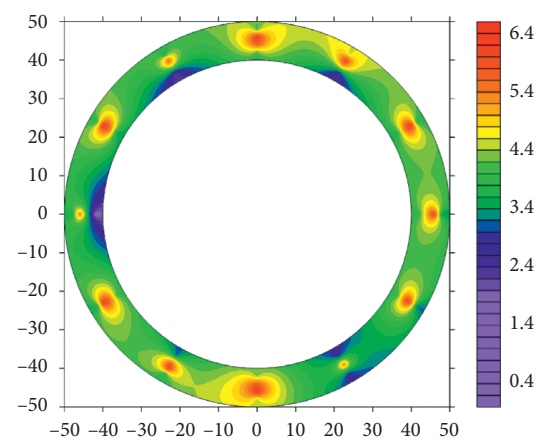

$\left(b_{2}\right)$ Section \#2

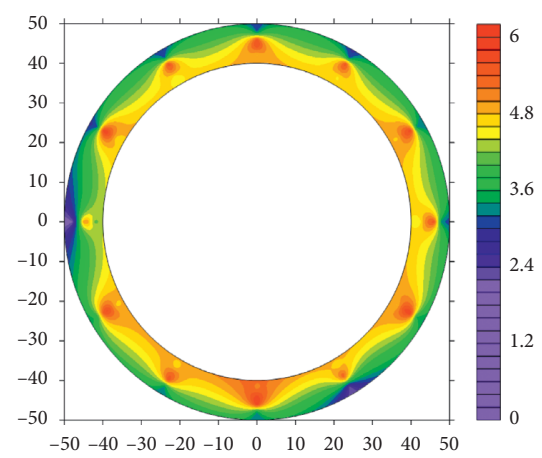

$\left(b_{5}\right)$ Section \#5

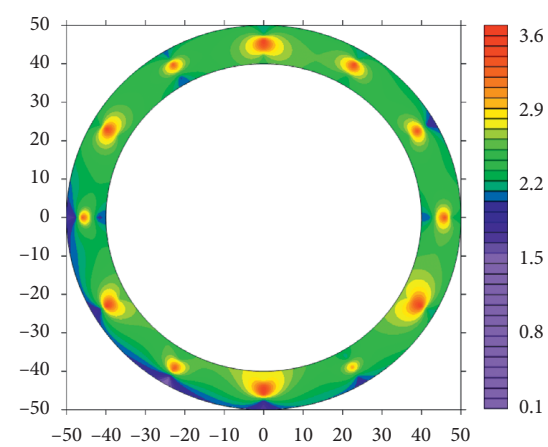

$\left(\mathrm{a}_{3}\right)$ Section \#3

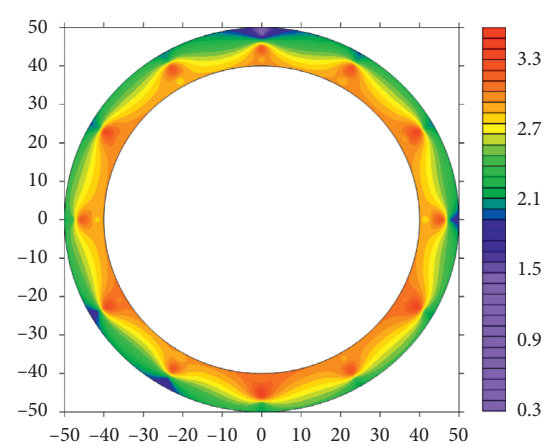

$\left(\mathrm{a}_{6}\right)$ Section $\# 6$

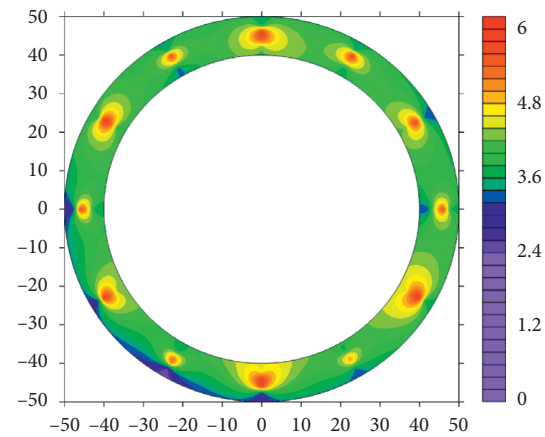

$\left(b_{3}\right)$ Section \#3

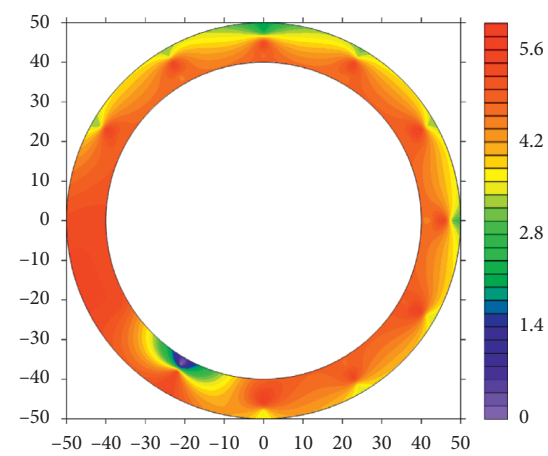

$\left(\mathrm{b}_{6}\right)$ Section \#6

(b)

Figure 9: Contour map of axial velocity of annular gap flow. (a) $\operatorname{Re}_{1}=115,513$. (b) $\operatorname{Re}_{3}=192,522$.

the water flow near the wall of the pipe vehicle is affected not only by the viscous resistance but also by the pressure resistance caused by the flow separation [44].
In order to understand the distribution law of the axial velocity of the annular slit flow, the axial velocity values of the measuring points with different radii at the zero degree 


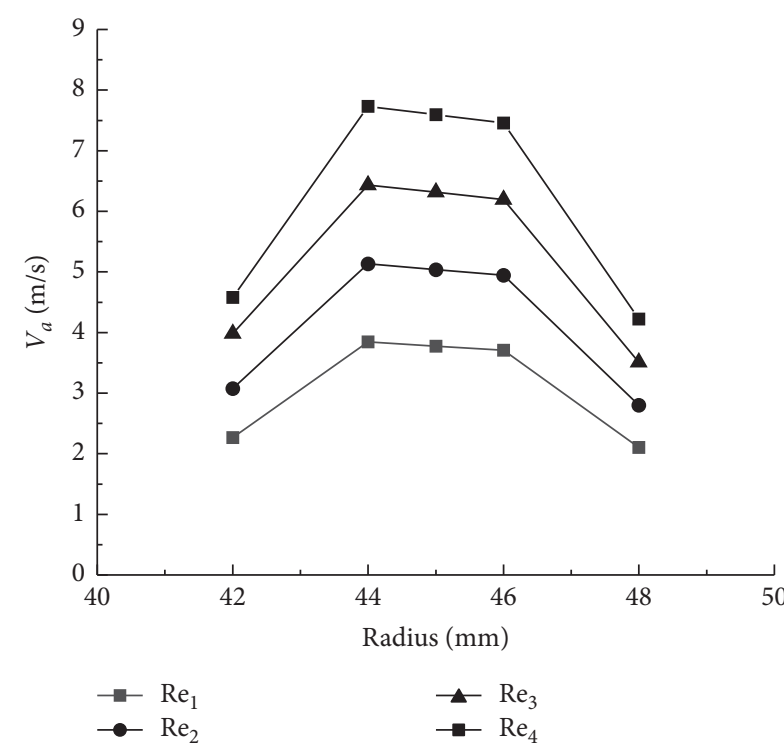

(a)

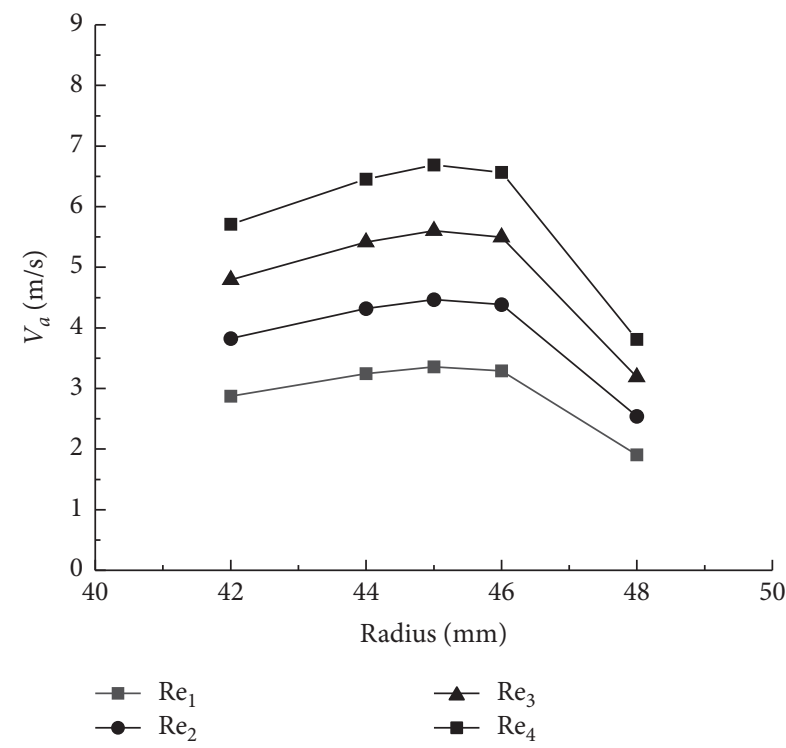

(c)

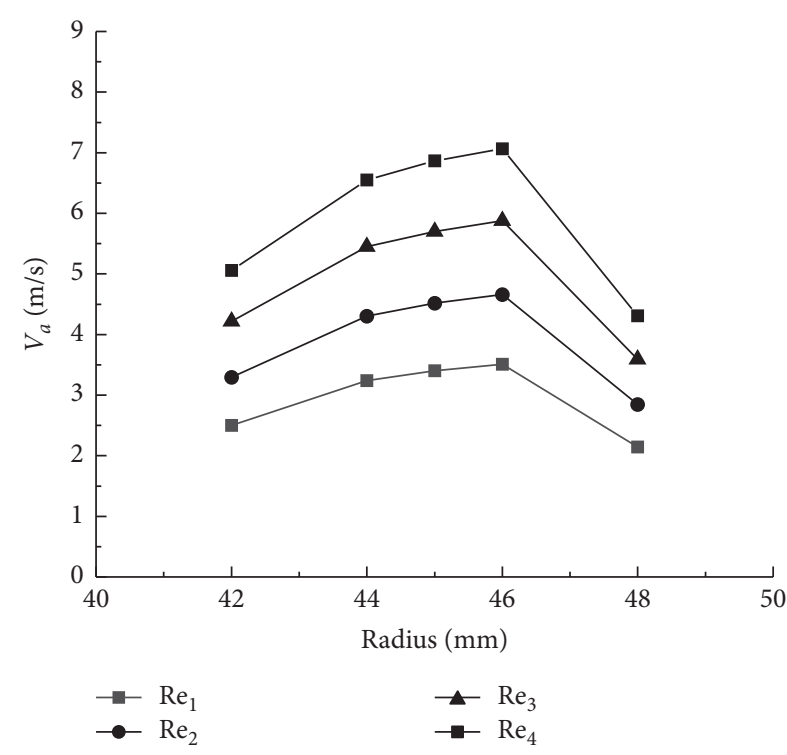

(b)

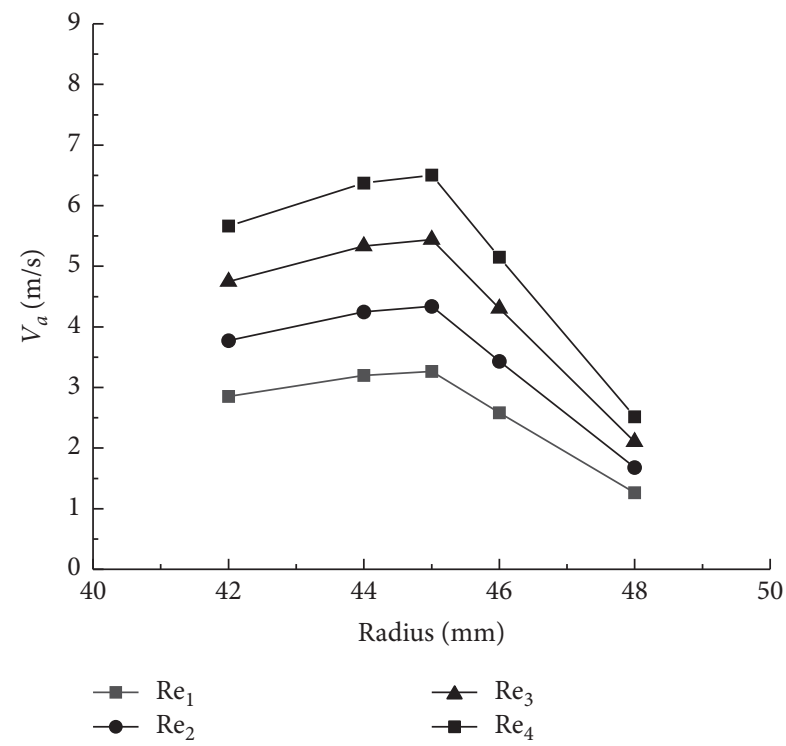

(d)

FiguRE 10: Schematic of axial flow velocity distribution of different cross sections. (a) Section \#1. (b) Section \#3. (c) Section \#5. (d) Section \#6.

position of each cross section are selected for analysis, as shown in Figure 10.

It can be seen from Figure 10 that the distribution law of annular gap flow under different Reynolds numbers is approximately the same. The axial flow velocity from the wall of the pipe vehicle to the wall of the pipeline first increases and then decreases, and the maximum value of the axial flow velocity is close to the pipeline wall. This is mainly due to the effect of viscous resistance; the axial flow velocity values near the wall of the pipe vehicle and the pipe wall are relatively low. As the distance from the pipe wall and the pipe vehicle wall increases, the effect of the viscous resistance gradually weakens and the water flow velocity increases. This phenomenon conformed to the theory of a viscous substratum
[45]. Since the influence of the pipe vehicle wall on the water flow is greater than that of the pipe wall, the maximum value of the axial flow velocity is biased toward the pipeline wall. It can also be seen from Figure 10 that the axial velocity in the annular gap is distributed in the form of a quadratic parabola, which is different from the logarithmic distribution of the velocity in the circular pipe. This shows that the pipe vehicle affects the distribution of water flow in the pipe.

4.1.2. Radial Velocity Distribution. The radial velocity points to the center of the circle along the diameter as positive and away from the center of the circle as negative. Take the Reynolds numbers $\mathrm{Re}_{1}=115,513$ and $\mathrm{Re}_{3}=192,522$ as 


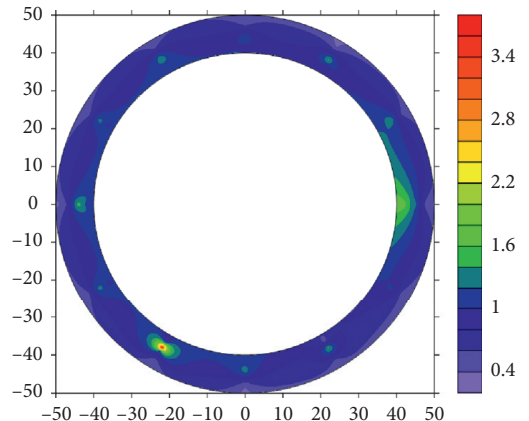

$\left(a_{1}\right)$ Section \#1

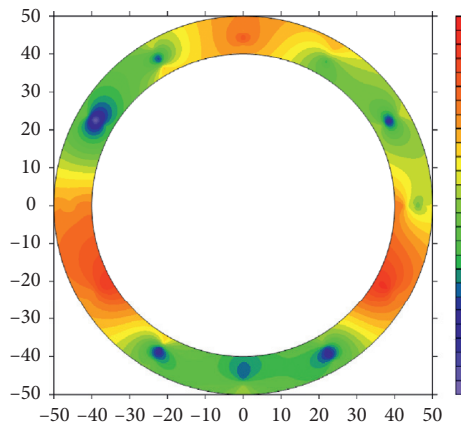

$\left(a_{4}\right)$ Section \#4

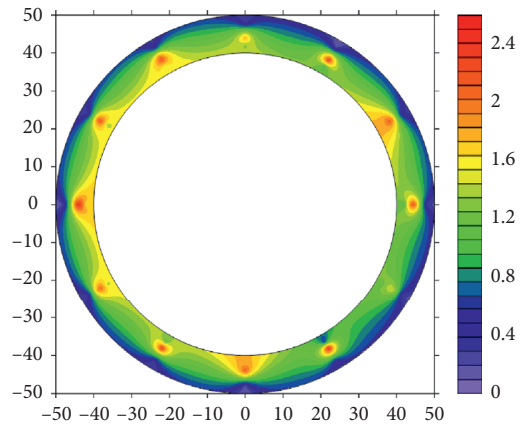

$\left(\mathrm{b}_{1}\right)$ Section \#1

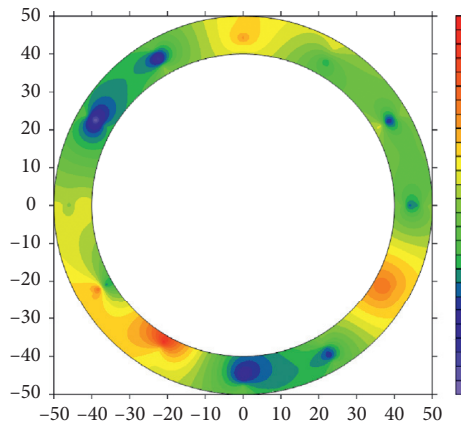

$\left(\mathrm{b}_{4}\right)$ Section \#4

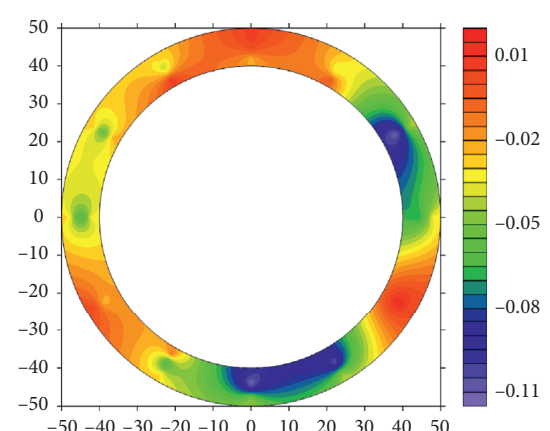

$\left(\mathrm{a}_{2}\right)$ Section \#2

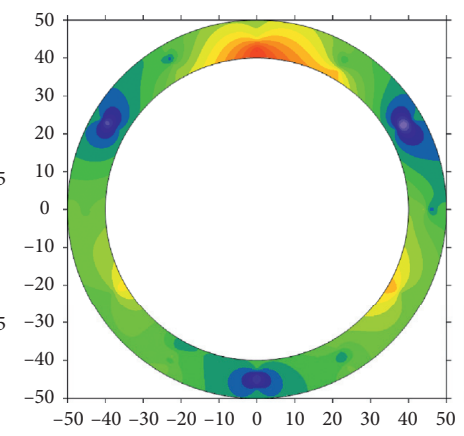

$\left(a_{5}\right)$ Section \#5

(a)

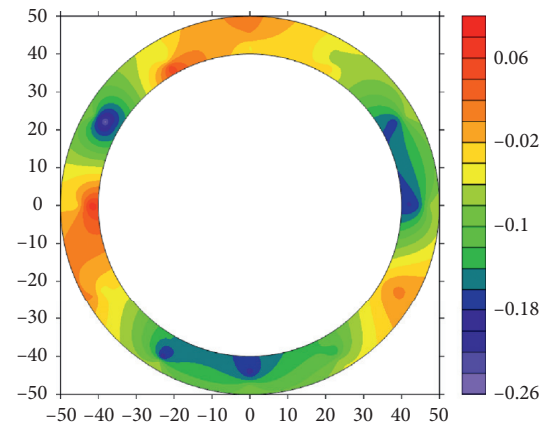

$\left(b_{2}\right)$ Section \#2

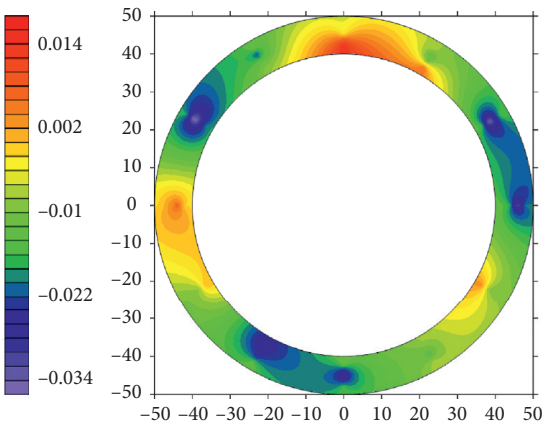

$\left(\mathrm{b}_{5}\right)$ Section \#5

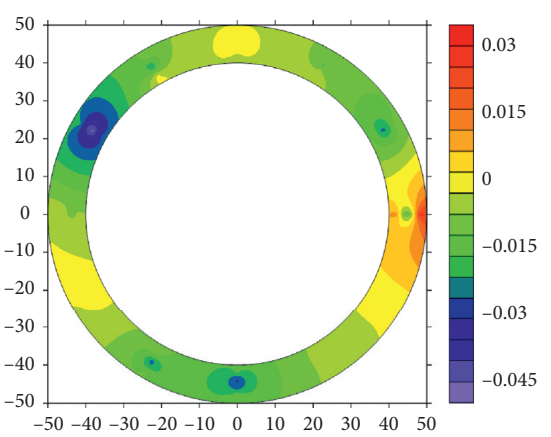

$\left(a_{3}\right)$ Section \#3

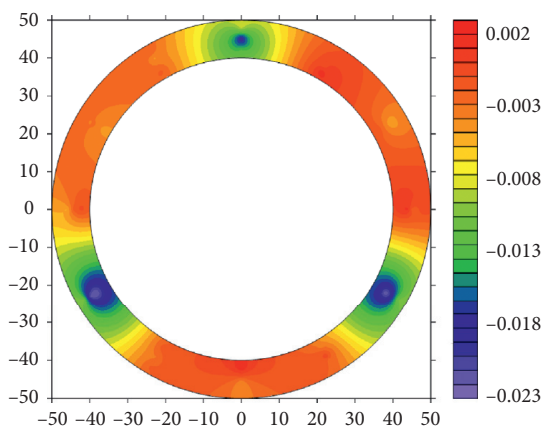

(a $a_{6}$ Section \#6

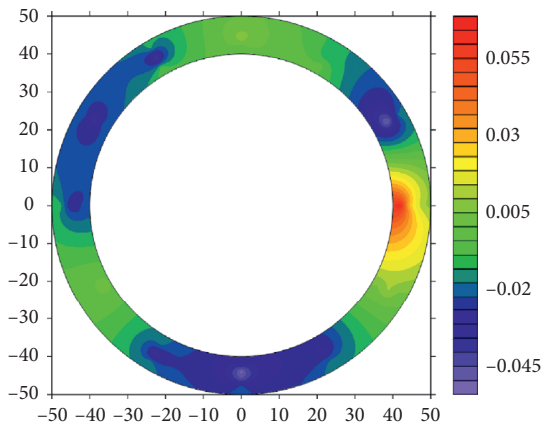

$\left(b_{3}\right)$ Section \#3

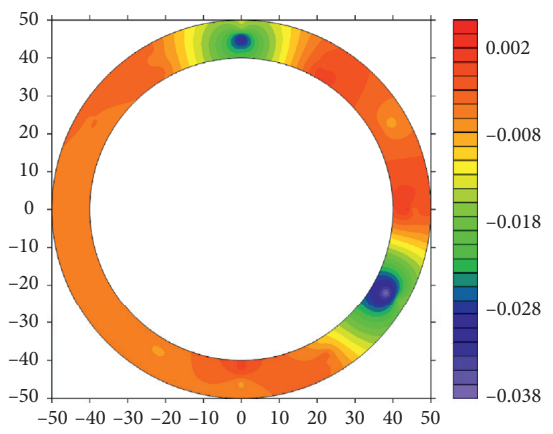

$\left(\mathrm{b}_{6}\right)$ Section \#6

(b)

Figure 11: Contour map of radial velocity of annular gap flow. (a) $\operatorname{Re}_{1}=115,513$. (b) $\operatorname{Re}_{3}=192,522$.

examples to analyze the radial velocity distribution. Contour maps of the radial velocity at different Reynolds numbers are shown in Figure 11.

It can be seen from Figure 11 that the radial velocity of the annular gap flow gradually increases with the increase in the Reynolds number. The radial velocity value reaches the maximum at Section \#1, while the radial velocity values of the other sections are much smaller. This is because Section $\# 1$ is located at the entrance of the annular gap. When the water flow changes from a full pipe flow to an annular gap 


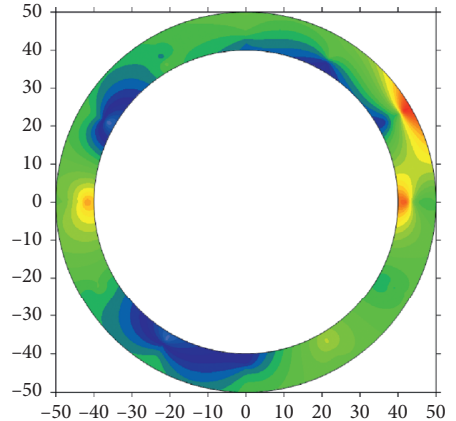

$\left(a_{1}\right)$ Section \#1

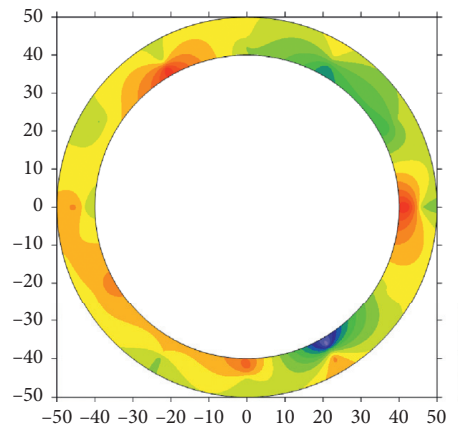

$\left(a_{4}\right)$ Section \#4

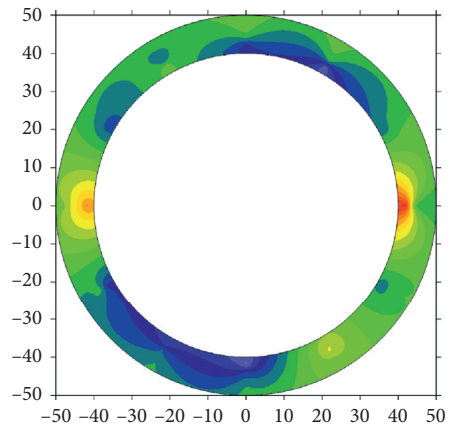

$\left(b_{1}\right)$ Section \#1

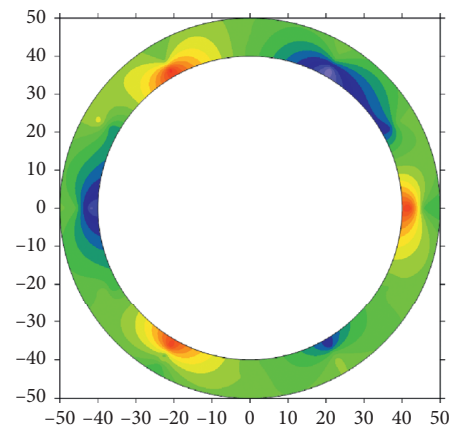

$\left(\mathrm{b}_{4}\right)$ Section \#4
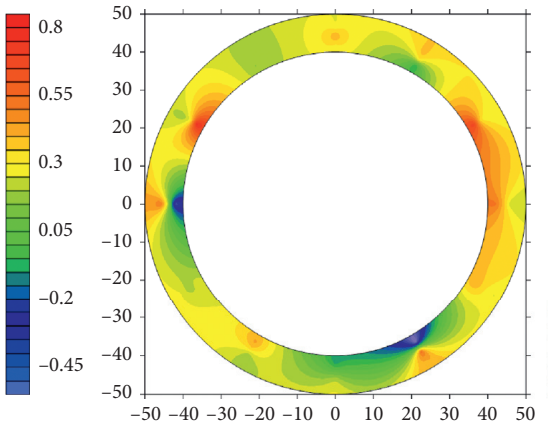

$\left(a_{2}\right)$ Section $\# 2$
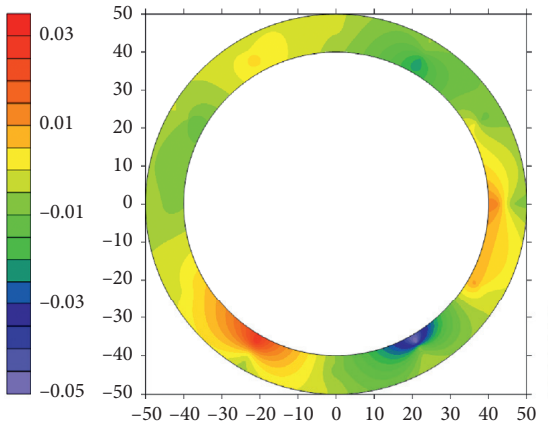

$\left(a_{5}\right)$ Section \#5

(a)
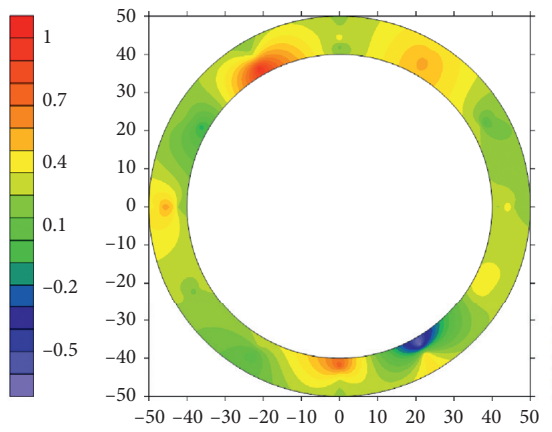

$\left(\mathrm{b}_{2}\right)$ Section \#2
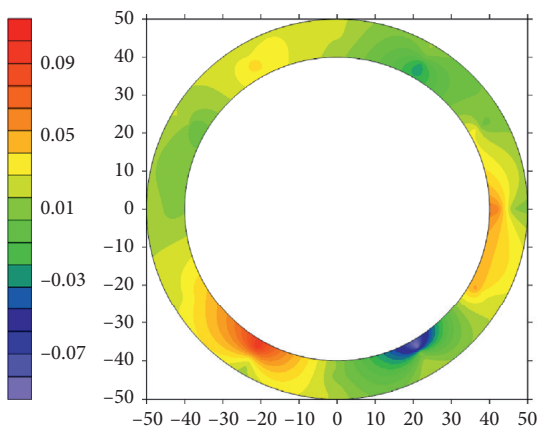

$\left(b_{5}\right)$ Section \#5
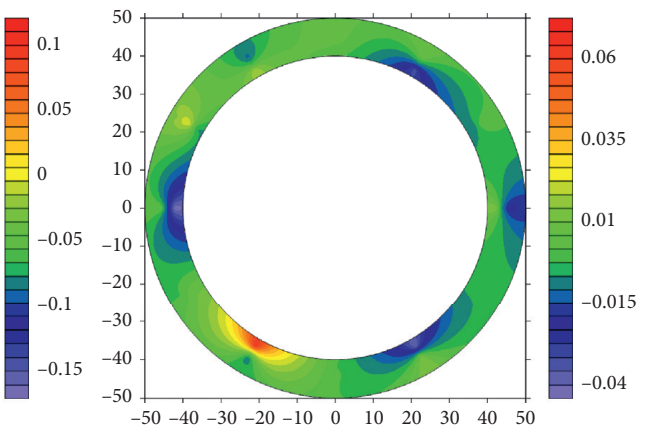

$\left(a_{3}\right)$ Section $\# 3$
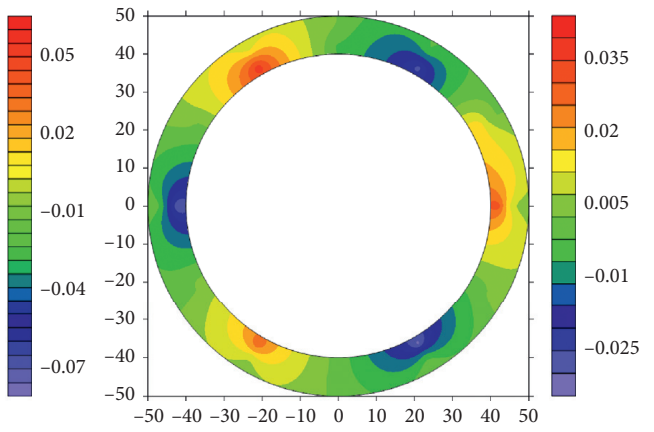

(a) Section \#6

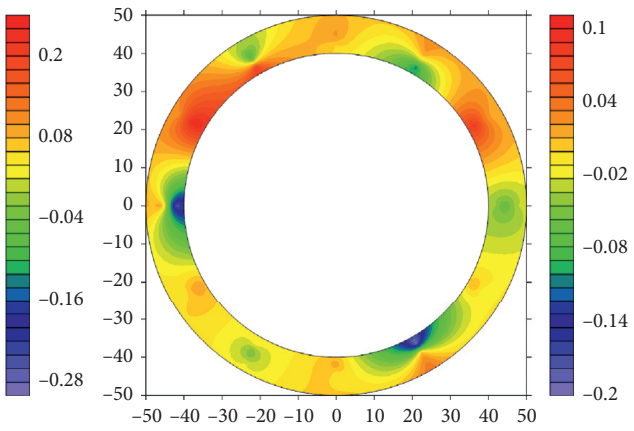

$\left(b_{3}\right)$ Section \#3
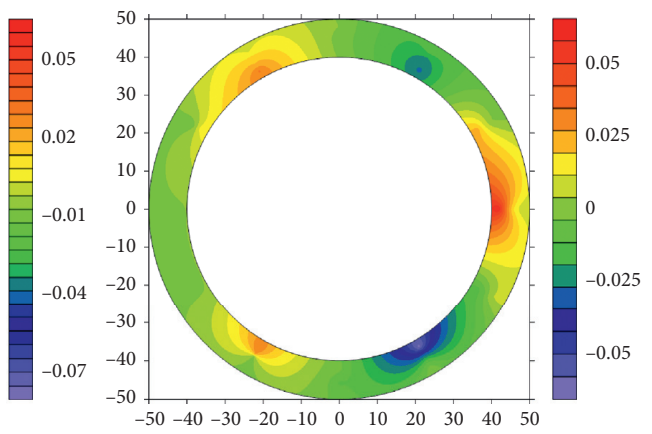

$\left(\mathrm{b}_{6}\right)$ Section \#6

(b)

FIgURE 12: Contour map of circumferential velocity of annular gap flow. (a) $\operatorname{Re}_{1}=115,513$. (b) $\operatorname{Re}_{3}=192,522$.

flow, the cross section suddenly shrinks and the water flow at the center of the pipe is squeezed toward the annular gap. Therefore, the water flow has a tendency to move toward the pipe wall, and the radial velocity value increases accordingly. After the water flow enters the inside of the annular gap, the flow state is gradually stabilized by the constraint of the annular gap space, and the flow line is also parallel to the axis of the pipe. Therefore, the partial velocity along the pipe diameter direction becomes smaller, that is, the radial flow velocity becomes smaller. Similar to the axial velocity, the 


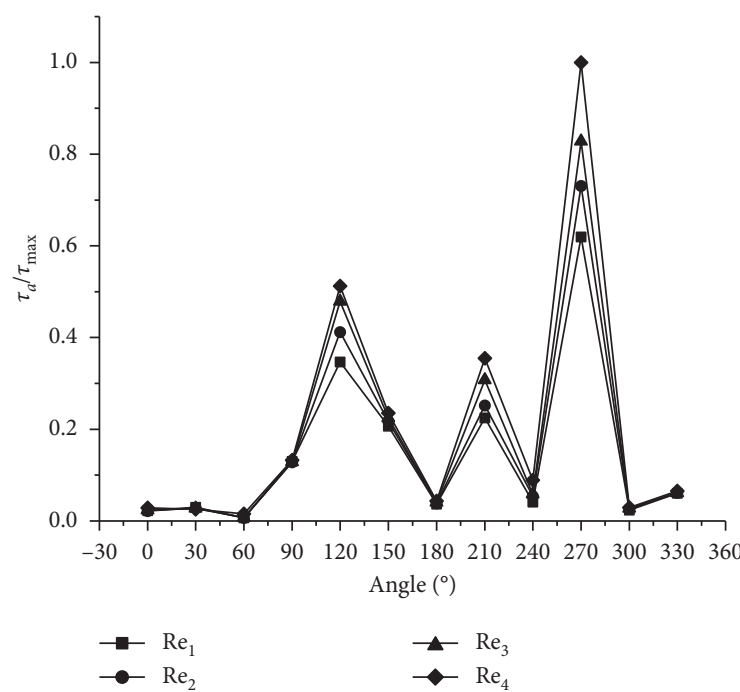

(a)

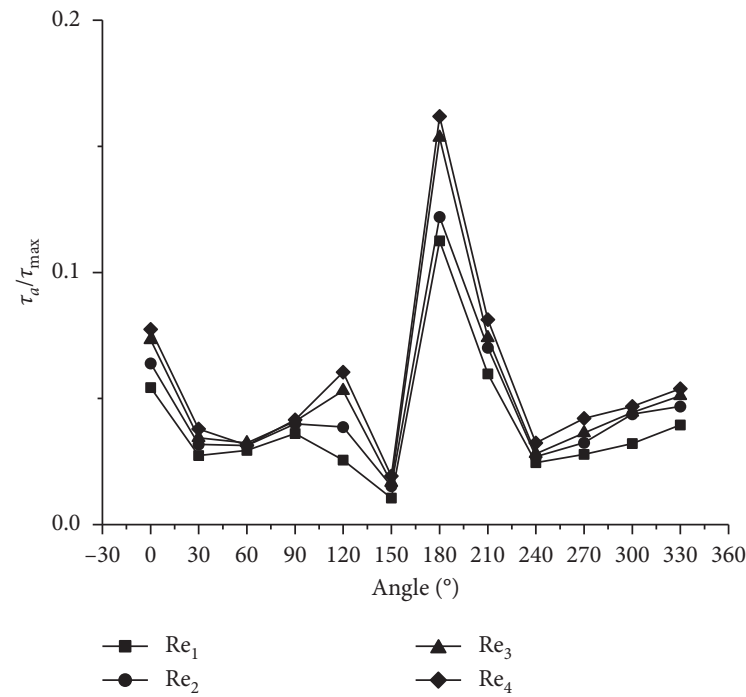

(c)

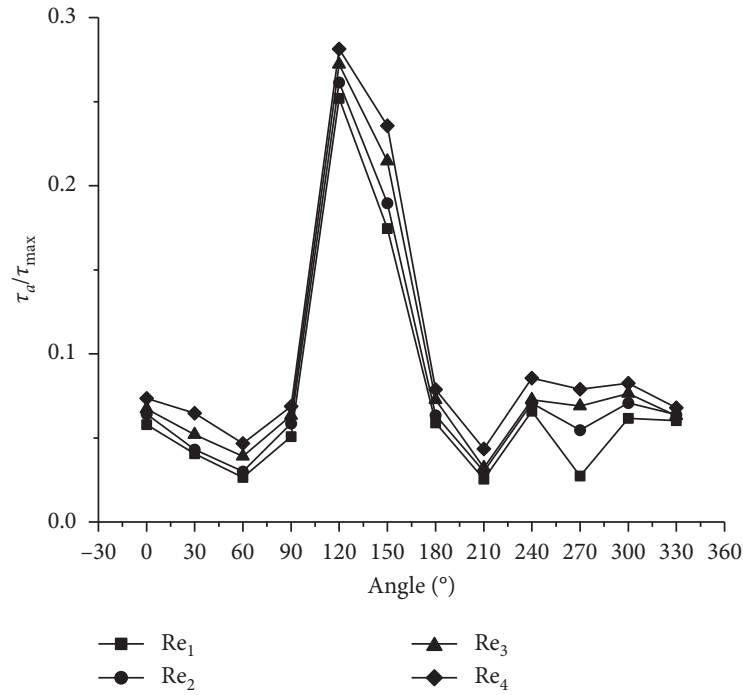

(e)

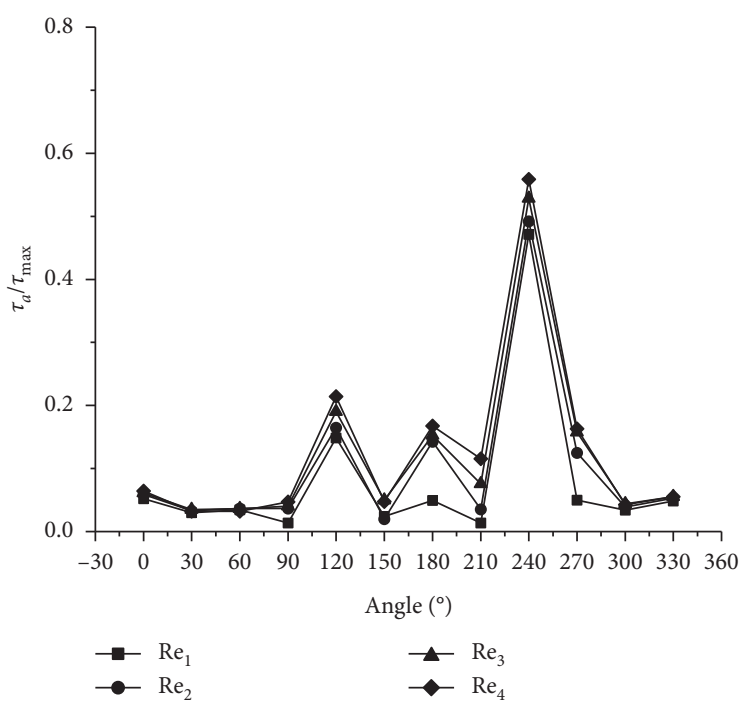

(b)

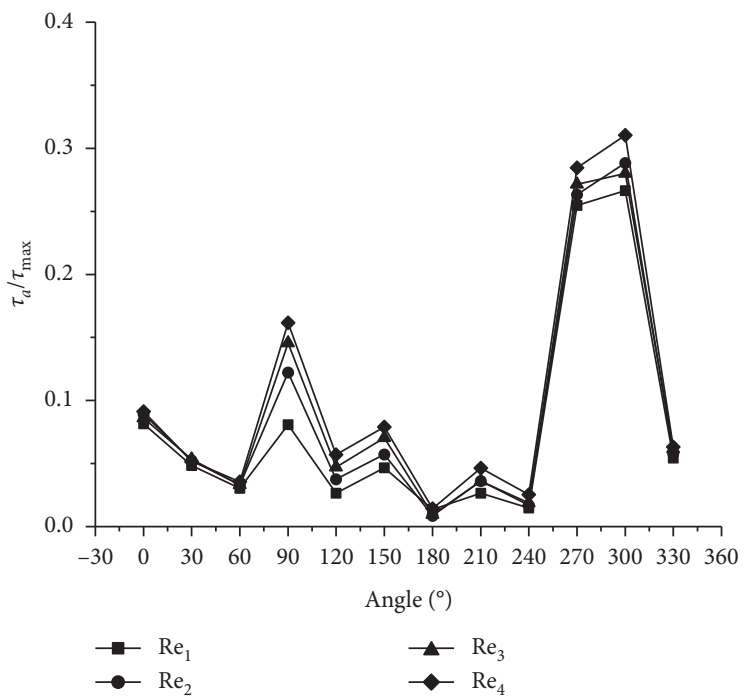

(d)

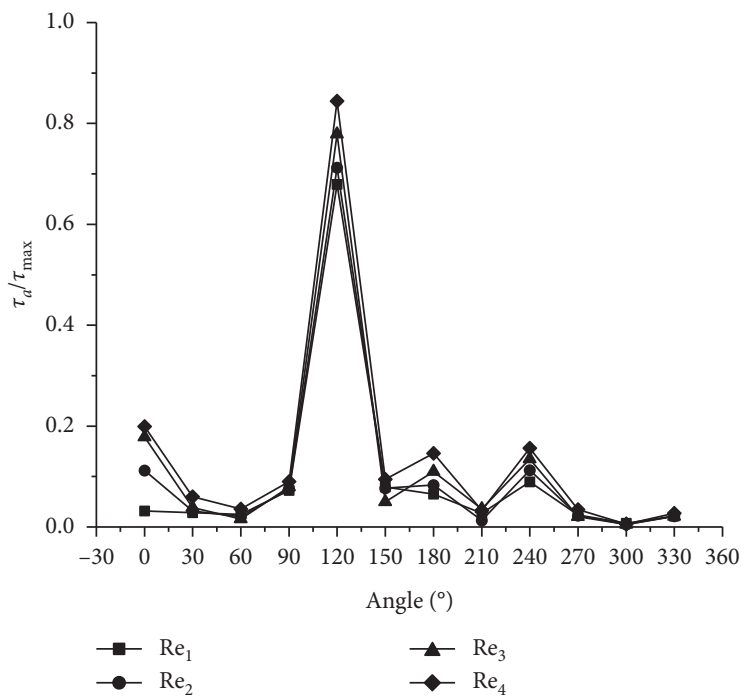

(f)

FIGURE 13: Wall axial shear stress distribution. Note: $\tau_{a}$ represents the wall axial shear stress of the point; $\tau_{\max }$ represents the maximum wall shear stress measured by the experiment. (a) Section \#1. (b) Section \#2. (c) Section \#3. (d) Section \#4. (e) Section \#5. (f) Section \#6. 


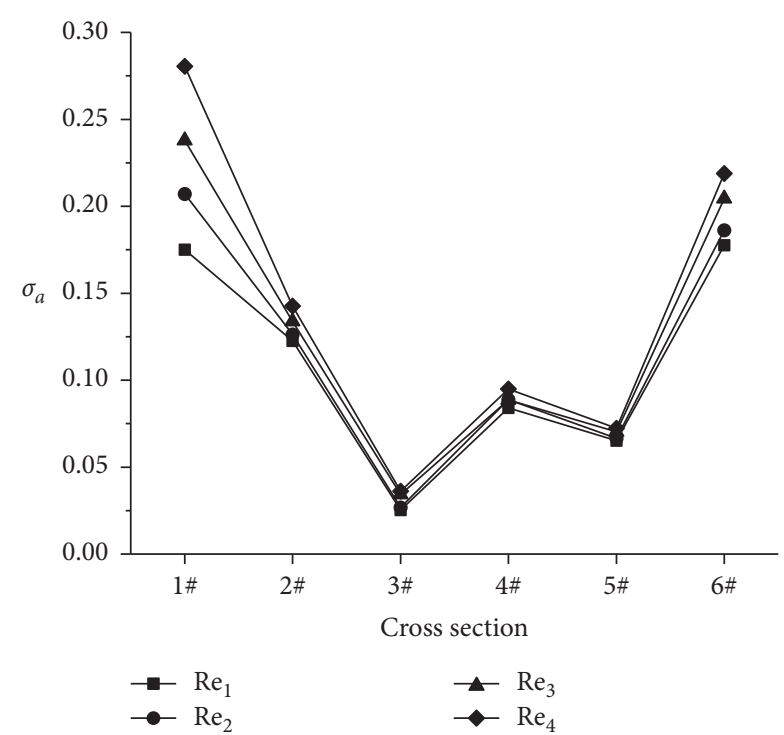

FIgURE 14: Schematic of standard deviation of wall axial shear stress.

velocity gradient near the wall of the pipe vehicle increases gradually as the Reynolds number increases. When the Reynolds number increases, the instability of the radial velocity in the annular gap also gradually increases, and the fluctuation of the radial velocity in the same section gradually increases. According to the color changes of the contour map, the radial velocity value is larger at the middle of the annular gap and gradually decreases toward the wall of the pipe vehicle and the wall of the pipe. The radial velocity value of each section is smaller than the axial velocity value, and the radial velocity value is positive or negative, which indicates that the radial velocity is directed in two directions: toward the circle's center and away from the circle's center along the pipe radius.

4.1.3. Circumferential Velocity Distribution. The circumferential velocity is positive in the counterclockwise direction along the tangent to the circumference and negative in the clockwise direction. Take the Reynolds numbers $\mathrm{Re}_{1}=115,513$ and $\mathrm{Re}_{3}=192,522$ as examples to analyze the circumferential velocity distribution.

It can be seen from Figure 12 that as the Reynolds number increases, the circumferential velocity value of each section also gradually increases. The circumferential flow velocity is similar to the radial flow velocity, and the flow velocity value reaches a maximum at Section \#1. Moreover, the fluctuation of the circumferential velocity value of the Section \#1 is also the most dramatic. This is because the end face of the pipe vehicle is located at Section \#1. When the water flows through the end face, it forms a flow around the cylinder and generates a large number of vortices. Section \#1 is closer to the support body, and the vortex formed at the support body diffuses to Section \#1, mixing with the vortex at this section, which further aggravates the distribution of the circumferential velocity of this section. Therefore, the value of the circumferential velocity at Section $\# 1$ is the largest and changes most drastically. After entering the annular gap, the circumferential velocity gradually becomes stable, and the velocity value also becomes smaller. There is a high-velocity region of circumferential velocity near the wall of the pipe vehicle, that is, the velocity gradient near the wall of the pipe vehicle is large. As the Reynolds number increases, the contour of the circumferential flow velocity at the wall of the pipe vehicle becomes dense, that is, the flow velocity gradient becomes larger. The increase in the Reynolds number also leads to an increase in the instability of the circumferential flow velocity in the annular gap, and the fluctuation of the circumferential flow velocity increases accordingly. Compared with the axial velocity value, the circumferential velocity value is smaller, that is, the partial velocity value of the annular gap flow along the circumferential tangent direction is smaller. Positive and negative circumferential velocity values indicate that the circumferential velocity is clockwise and counterclockwise along the circumferential tangent of the section.

4.2. Analysis of Wall Shear Stress of the Cylinder Pipe Vehicle. The wall shear stress acting on the cylinder pipe vehicle can be divided into axial and circumferential directions. The wall axial shear stress coincides with the axis of the pipeline. Similar to the annular gap flow, it is positive along the direction of the flow, but negative otherwise. The wall circumferential shear stress direction is the same as the circumferential tangent direction. It is defined as positive in the counterclockwise direction along the circumference and negative in the clockwise direction.

4.2.1. Distribution of Wall Axial Shear Stress. The wall axial shear stress distribution of each section under different Reynolds number conditions is shown in Figure 13. The wall axial shear stress is expressed in a dimensionless form.

It can be seen from Figure 13 that (1) The wall axial shear stress shows a gradually increasing trend with the increase in Reynolds number. The water flow in the pipeline is turbulent due to the Reynolds number being greater than 2000. There is a thin layer of viscous bottom layer close to the solid side wall in turbulence. According to the previous analysis of the article, it can be seen that as the Reynolds number increases, the velocity gradient near the wall of the pipe vehicle and the wall axial shear stress also increase. The increase in the wall axial shear stress in turn affects the surrounding fluid, making the fluid instability gradually increase. (2) The overall change trend of the wall axial shear stress on the circumference of the same section is similar under different Reynolds numbers. When the Reynolds number is constant, the variation of the wall axial shear stress on the circumference of each section is quite different. This is mainly due to the difference in the change in axial flow velocity of each section. The change in the flow velocity also makes the velocity gradient near the wall surface of the pipe vehicle different. So, the corresponding changes in the axial shear stress of each section are also different. (3) The wall axial shear stress is most unstable at Sections \#1 and 6, and the fluctuation range of the wall axial shear stress value is large. 


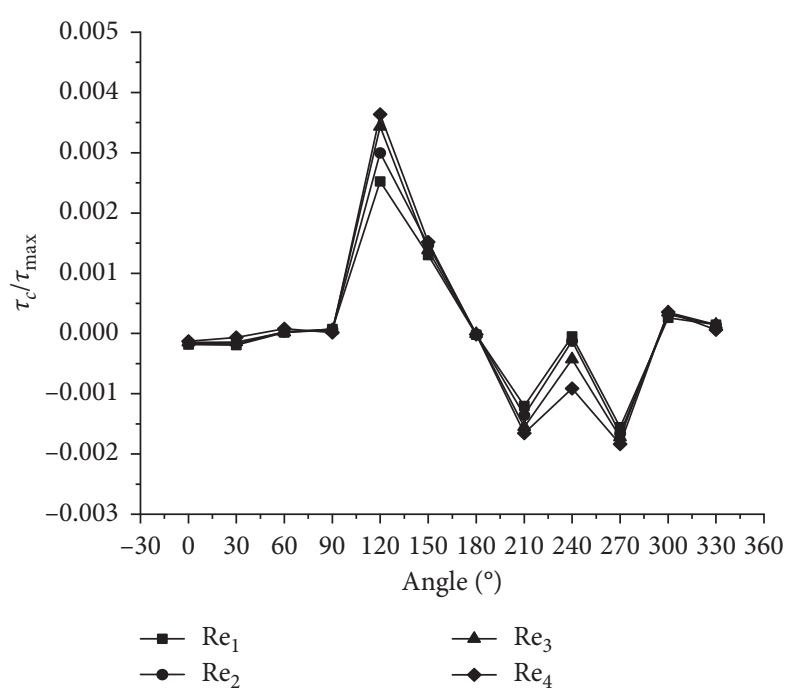

(a)

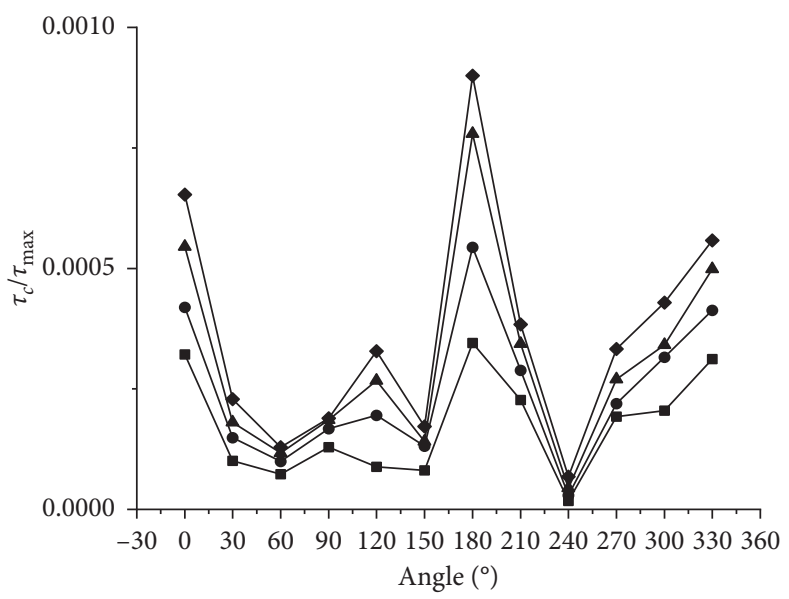

(c)

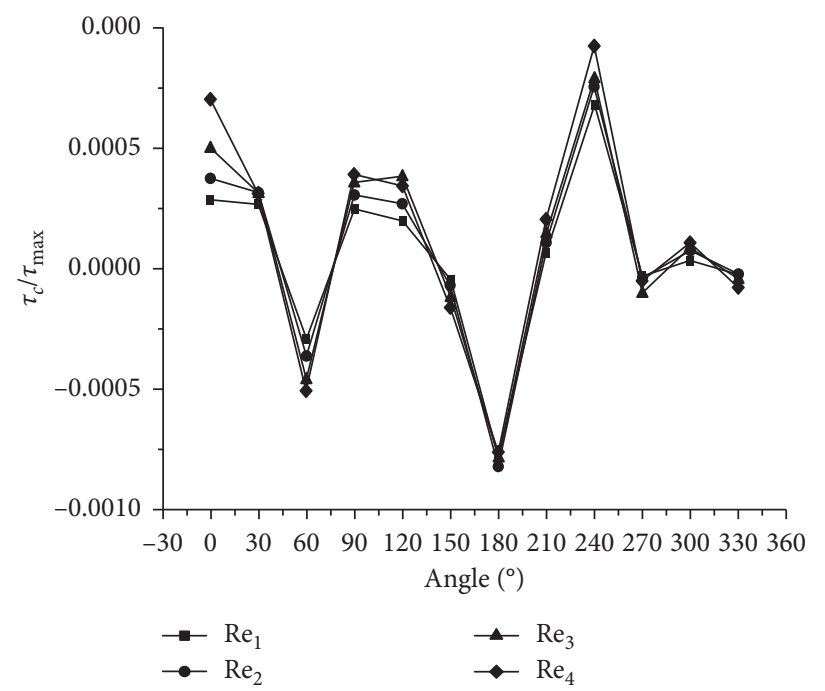

(e)

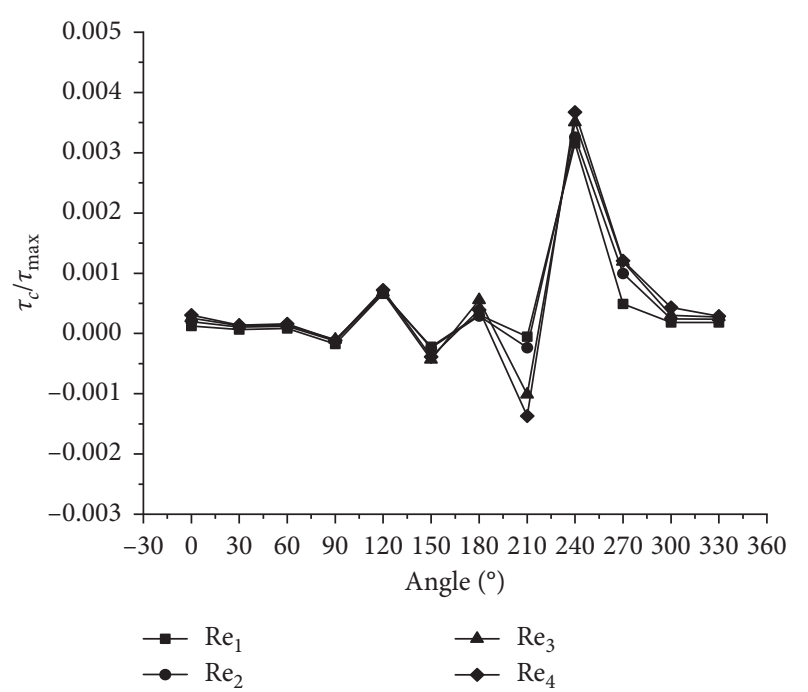

(b)

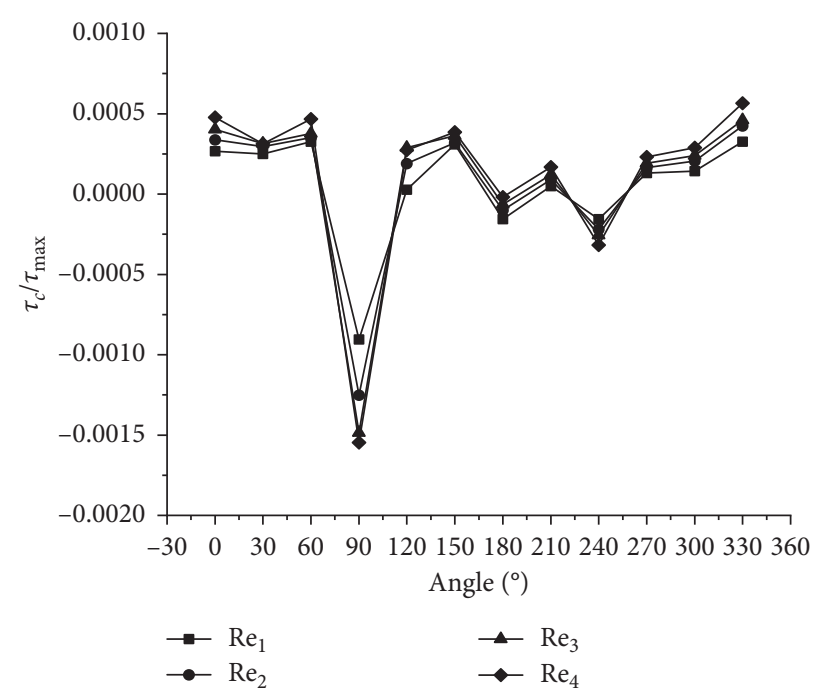

(d)

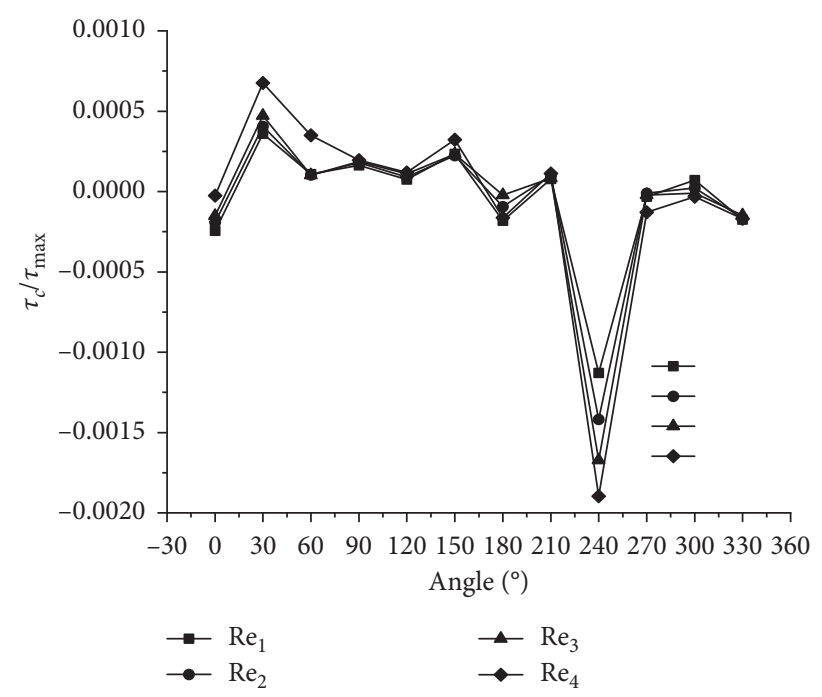

(f)

FIgURe 15: Wall circumferential shear stress distribution. Note: $\tau_{c}$ represents the wall circumferential shear stress of the point; $\tau_{\text {max }}$ represents the maximum wall shear stress measured by the experiment. (a) Section \#1. (b) Section \#2. (c) Section \#3. (d) Section \#4. (e) Section \#5. (f) Section \#6. 


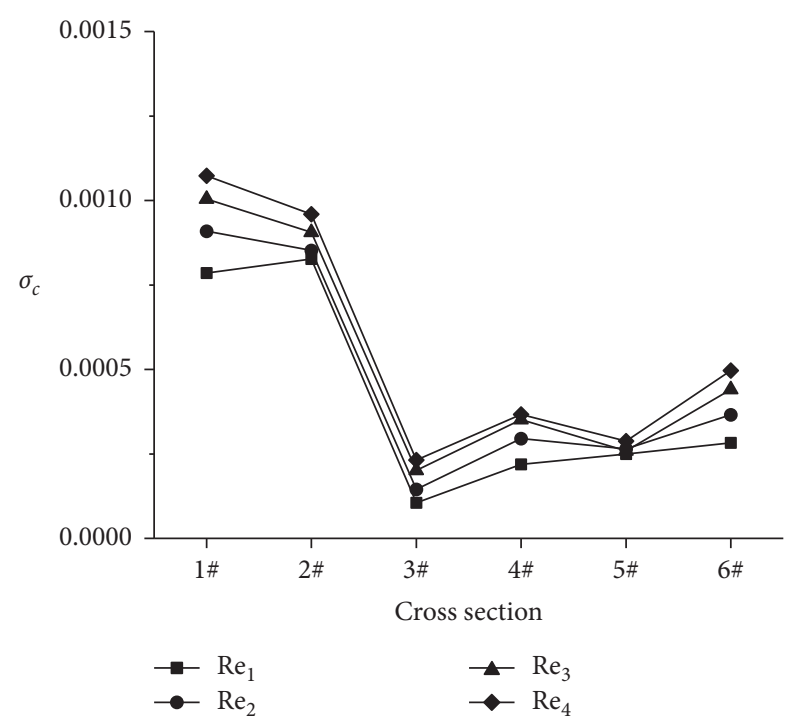

FIGURE 16: Schematic of standard deviation of wall circumferential shear stress.

According to the analysis of the flow field in the article, part of the water flow is squeezed toward the wall of the pipe vehicle when entering the annular gap. Therefore, the interaction between the water flow and the wall of the pipe vehicle is enhanced. There are more vortices at Section \#1, which further enhances the interaction between the water flow and the wall of the pipe vehicle. So, the axial shear stress of Section \#1 is the most unstable and the fluctuation range is the largest. At the exit of the annular gap, the streamline bends due to the influence of the end face of the pipe vehicle, and the interaction between the water flow and wall of the pipe vehicle is increased. The backflow of part of the water flow at Section \#6 makes the flow pattern change more drastically. Therefore, the wall axial shear stress of Section \#6 is large and changes drastically. (4) Compared with Sections \# 1 and 6, the wall axial shear stress value and variation fluctuation range of each cross section within the annular gap are smaller. This is because the value of the axial flow velocity in the annular gap becomes smaller, and the flow velocity gradient near the wall of the pipe vehicle decreases. The axial flow velocity distribution in the annular gap is relatively stable and the fluctuation is small. So, the corresponding wall axial shear stress value and the fluctuation are small. (5) It can also be seen from Figure 13 that the ratios of $\tau_{\mathrm{a}} / \tau_{\max }$ are all positive, which indicates that the direction of wall axial shear stress is along the water flow.

The standard deviation $\sigma_{a}$ is used to reflect the degree of dispersion of the axial shear stress at all points on each section under different Reynolds numbers. The calculation formula of the standard deviation $\sigma_{a}$ is as follows:

$$
\sigma_{a}=\sqrt{\frac{1}{n} \sum_{i=1}^{n}\left(\tau_{a i}-\overline{\tau_{a}}\right)^{2}},
$$

where $n$ represents the total number of measuring points on a section, $\tau_{a i}$ represents the axial shear stress value of each measuring point, and $\overline{\tau_{\mathrm{a}}}$ represents the average wall axial shear stress on the section.

Figure 14 shows the variation of the standard deviation of the wall axial shear stress of each section under different Reynolds numbers. It can be seen from the figure that as the Reynolds number increases, the degree of dispersion of the wall axial shear stress in each section also shows a gradually increasing trend. The result indicates that increasing the Reynolds number will reduce the stability of the wall axial shear stress of the pipe vehicle. The instability of the wall shear stress affects the annular gap flow again, so the stability of the annular gap flow decreases. When the Reynolds number is unchanged, the standard deviations of Sections \#1 and 6 are larger, that is, the wall axial shear stress value of all measuring points on these two cross sections fluctuates most drastically, which is consistent with the previous analysis. However, the wall axial shear stress dispersion of the other sections is relatively small, that is, the fluctuation of the wall axial shear stress at other sections in the annular gap is small. It can be seen that the end structure of the pipe vehicle has a great influence on the fluctuation of the wall shear stress. It is possible to reduce this fluctuation by optimizing the structure of the pipe vehicle and improving the stability of the wall shear stress.

\subsubsection{Distribution of Wall Circumferential Shear Stress.} The wall circumferential shear stress distribution of each cross section under different Reynolds number is shown in Figure 15.

Similar to the wall axial shear stress, the wall circumferential shear stress of each section increases as the Reynolds number increases. According to equation (8), there is a positive correlation between wall shear stress and water flow rate. From the previous analysis, we know that when the Reynolds number increases, the circumferential flow velocity also increases, so the wall circumferential shear stress becomes larger. The overall trend of the wall circumferential shear stress along the same cross section circumference is the same under different Reynolds numbers, but the variation of wall circumferential shear stress in each section is different. The wall circumferential shear stress values of Sections \#1 and 2 change drastically and are larger than those of the other sections under the same Reynolds number. This is because the circumferential velocity of the water flow at Sections \#1 and 2 has a large variation and the circumferential velocity at the two sections is large, so the wall circumferential shear stress values of Sections \# 1 and 2 become larger. The wall circumferential shear stress values of Sections \#3-6 sections are small, and the fluctuation range of the wall circumferential shear stress values of these sections is also small. This is mainly because the water flow gradually stabilizes after entering the annular gap, and the interaction between the water flow and the wall of the pipe vehicle is no longer intense, so the wall circumferential shear stress values of these sections are relatively small. Compared with the wall axial shear stress, the wall circumferential shear stress is much smaller. This is because there is a corresponding relationship between wall shear stress and flow velocity. Since 


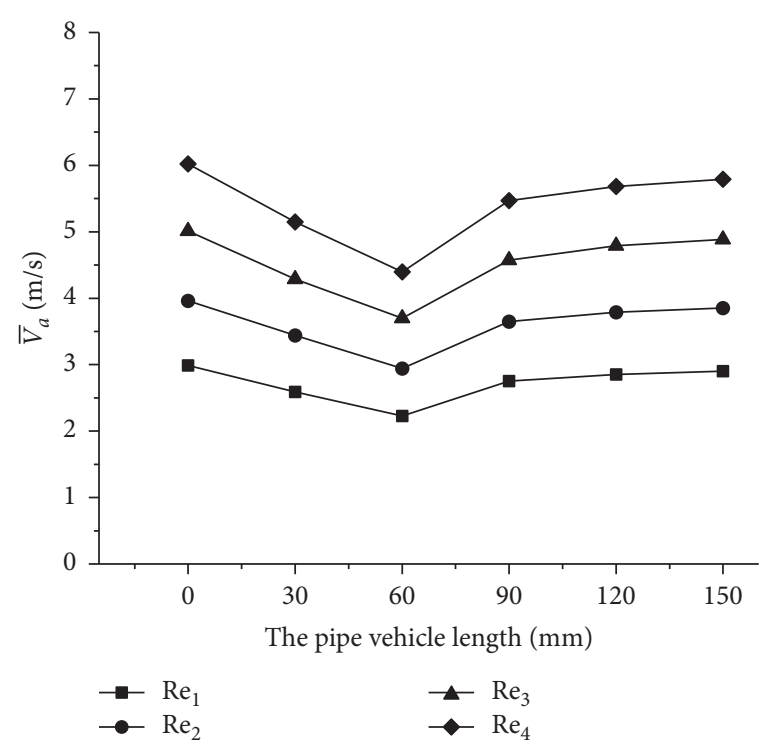

(a)

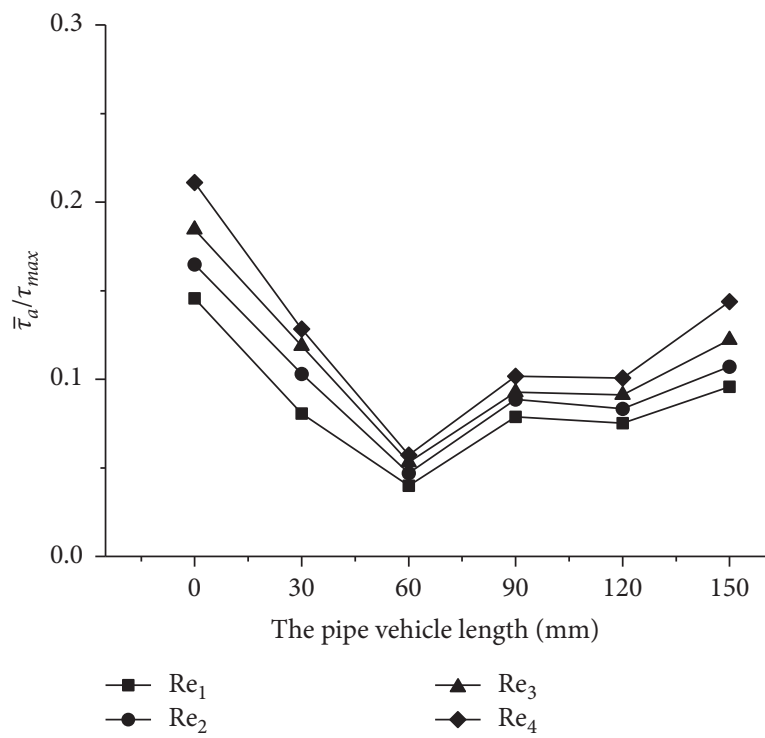

(c)

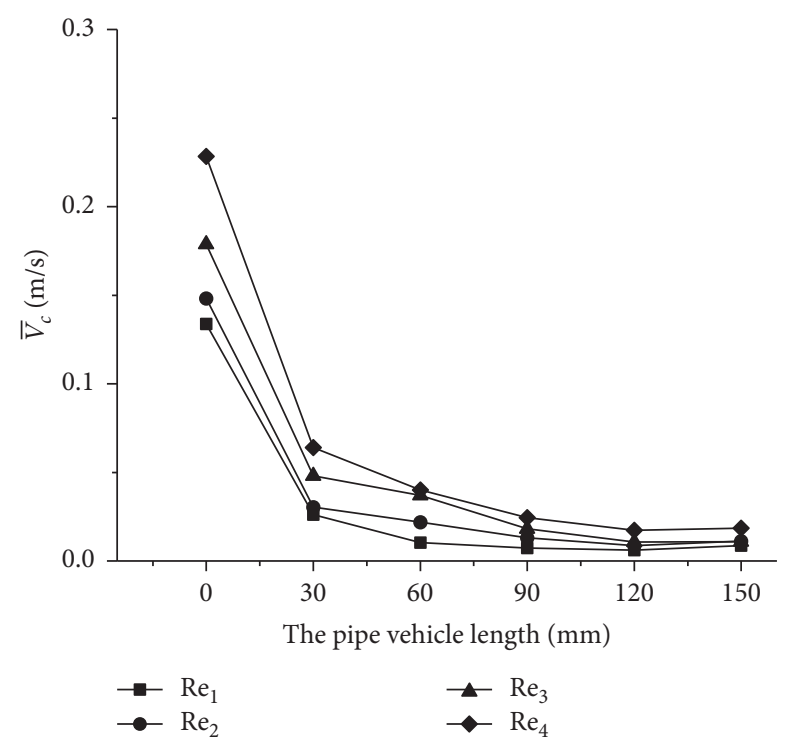

(b)

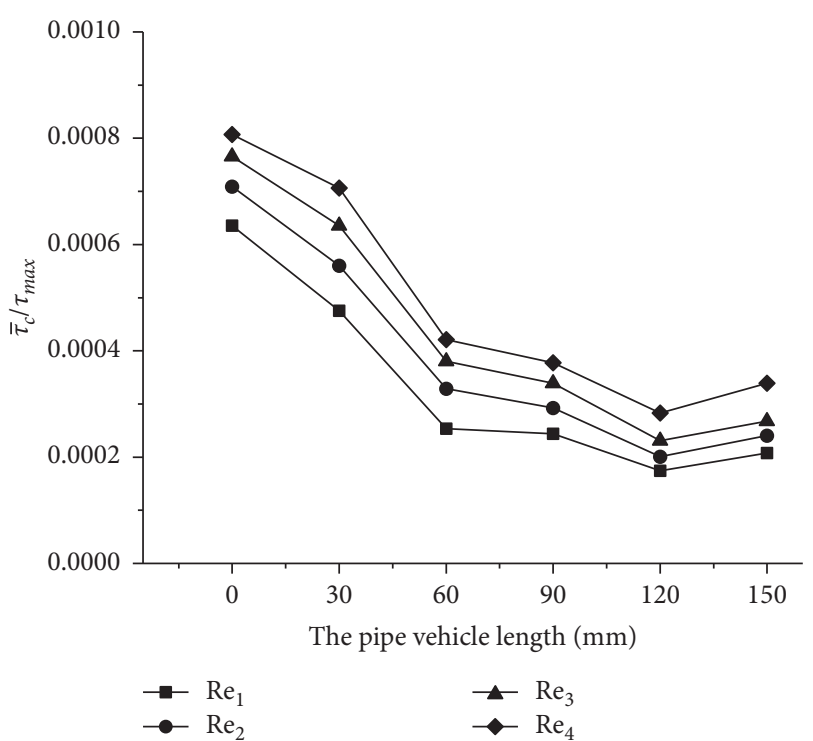

(d)

Figure 17: The distribution of wall shear stress and velocity along the length of the cylinder pipe vehicle. (a) The distribution of wall axial average shear stress. (b) The distribution of wall circumferential average shear stress. (c) The distribution of axial average velocity. (d) The distribution of circumferential average velocity. Note: the ordinate is the absolute value of the wall circumferential shear stress and flow velocity in (b) and (d).

the axial flow velocity is greater than the circumferential flow velocity, the corresponding wall axial shear stress is greater than the circumferential shear stress. The result indicates that the angle between the shear stress and the axis of the pipe is relatively small, that is, the wall shear stress direction almost coincides with the pipe axis. Positive and negative wall circumferential shear stress values indicate that the direction is clockwise or counterclockwise along the circumference, respectively.

The standard deviation $\sigma_{c}$ is used to reflect the fluctuation of the wall circumferential shear stress of each section under different Reynolds numbers, as shown in Figure 16. The calculation of $\sigma_{c}$ refers to equation (20).
It can be seen from Figure 16 that the standard deviations of Sections \#1 and 2 are relatively large and those for the other sections are relatively small. This shows that the wall circumferential shear stress values of the measuring points on the two cross sections are relatively dispersed. This is mainly due to the large fluctuations in the circumferential velocity of the two sections, so the corresponding wall shear stress fluctuations also increase. As the Reynolds number increases, the standard deviation of the wall circumferential shear stress also gradually increases. This shows that the change in the Reynolds number has an influence on the instability of the wall circumferential shear stress. As the Reynolds number increases, this instability gradually 
increases. The instability of the wall circumferential shear stress reacts to the annular gap flow, which increases the fluctuation of the circumferential velocity. Therefore, the fluctuation of the wall circumferential shear stress can be reduced by optimizing the structure of the pipe vehicle.

4.3. Distribution of Wall Shear Stress and Velocity along the Length of Cylinder Pipe Vehicle. In order to further study the relationship between the wall shear stress and the annular gap velocity, the curves of the average shear stress and the average velocity in the length direction of the pipe vehicle body are shown in Figure 17.

It can be seen from Figure 17 that both the annular gap flow and the wall shear stress are affected by the Reynolds number, both of which increase as the Reynolds number increases. The wall axial average shear stress and axial average flow velocity are correlated: both show a trend of first decreasing and then increasing along the direction of the pipe vehicle body. Both of them reach the minimum when the length of the pipe vehicle body is $60 \mathrm{~mm}$. However, the wall circumferential average shear stress and the circumferential average flow velocity generally show a gradually decreasing trend along the length of the pipe vehicle body but slightly increase at the end of the pipe vehicle body. The values of shear stress and annular gap flow velocity reach the maximum at the entrance of the annular gap. This shows that section shrinkage and the existence of support bodies have a great influence on the wall shear stress and annular gap flow. Therefore, the influence of the pipe vehicle on wall shear stress and annular gap flow can be reduced by optimizing the structure of the pipe vehicle.

\section{Conclusions}

The characteristics of the concentric annular gap flow and the wall shear stress under different Reynolds numbers were analyzed using the model experiment. The main conclusions are summarized as follows:

(1) As the Reynolds number increases, the flow velocity of the annular slit flow gradually increases, but the flow stability decreases and the fluctuation of the velocity increases. The water flow at the entrance and exit of the annular gap changes the most drastically. The water flow inside the annular gap presents a quadratic parabolic distribution that first increases and then decreases along the pipeline vehicle wall to the pipe wall. The axial velocity is larger than the circumferential and radial velocity. The axial flow velocity is along the direction of the pipe axis, the radial flow velocity is directed to the center of the circle along the radius and away from the center of the circle, and the circumferential flow velocity is clockwise or counterclockwise along the circumferential tangent.

(2) As the Reynolds number increases, the value of the wall shear stress also shows a gradually increasing trend, while the stability of the shear stress gradually decreases. The change in the wall shear stress affects the stability of the annular gap flow. The axial shear stress reaches the maximum at the entrance of the annular gap. The wall axial shear stress value is much larger than the wall circumferential shear stress value. The axial shear stress is the same as the direction of the water flow, while the circumferential shear stress appears in two directions: clockwise or counterclockwise along the circumferential tangent.

(3) The change trends of wall average shear stress and average annular gap flow are consistent along the length of the pipe vehicle. The wall axial average shear stress and the axial average flow velocity show a trend of first decreasing and then increasing along the length of the pipe vehicle. The wall circumferential average shear stress and the circumferential average flow velocity generally show a gradually decreasing trend along the length of the pipe vehicle but slightly increase at the end of the pipe vehicle.

\section{Data Availability}

The data used to support the findings of this study are available from the corresponding author upon request.

\section{Conflicts of Interest}

The authors declare that there are no conflicts of interest regarding the publication of this paper.

\section{Authors' Contributions}

X.M. Jia and J.R. Song designed the experimental schemes and purchased the experimental materials; X.H. Sun provided the experimental sites and the necessary experimental equipment; X.H. Sun reviewed the manuscript and provided the technical guidance on the research subject; X.M. Jia wrote and revised the manuscript and completed the numerical calculation.

\section{Acknowledgments}

Supports from the Collaborative Innovation Center of New Technology of Water-Saving and Secure and Efficient Operation of Long-Distance Water Transfer Project at Taiyuan University of Technology, X.H Sun Province Supercomputing and Data Center, and X.H Sun Province Scientific Cloud Computing Center are acknowledged. This project was supported by the National Natural Science Foundation of China (grant nos. 51179116, 51109155, and 50579044) and the Natural Science Foundation of Shanxi Province (Grant nos. 2015011067 and 201701D221137).

\section{References}

[1] M. E. Charles, "Theoretical analysis of the concentric flow of cylindrical forms," Canadian Journal of Chemical Engineering, vol. 41, no. 2, pp. 46-51, 1963.

[2] H. S. Ellis, "The pipeline flow of capsules: part 3-an experimental investigation of the transport by water of single cylindrical and spherical capsules with density equal to that of 
the water," The Canadian Journal of Chemical Engineering, vol. 42 , no. 1, pp. 1-8, 1964.

[3] H. S. Ellis, "The pipeline flow of capsules part 4-an experimental investigation of the transport in water of single cylindrical "capsules" with density greater than that of the water," The Canadian Journal of Chemical Engineering, vol. 42 , no. 2, pp. 69-76, 1964.

[4] H. S. Ellis, "The pipeline flow of capsules part 5-an experimental investigation of the transport by water of single spherical capsules with density greater than that of the water," The Canadian Journal of Chemical Engineering, vol. 42, no. 4, pp. 155-161, 1964.

[5] H. S. Ellis and L. H. Bolt, "The pipeline flow of capsules: part 7-an experimental investigation of the transport by two oils of single cylindrical and spherical capsules with density equal to that of the oil," The Canadian Journal of Chemical Engineering, vol. 42, no. 5, pp. 201-206, 1964.

[6] G. F. Round and L. H. Bolt, "An experimental investigation of the transport in oil of single, denser than oil, spherical and cylindrical capsules," Canadian Journal of Chemical Engineering, vol. 41, no. 2, pp. 43-45, 1963.

[7] G. W. Hodgson and M. E. Charles, "The concept of capsule pipelining," Canadian Journal of Chemical Engineering, vol. 41, no. 2, pp. 43-45, 1963.

[8] R. Newton, P. J. Redberger, and G. F. Round, "Numerical analysis of some variables determining free flow," Canadian Journal of Chemical Engineering, vol. 42, pp. 168-173, 1963.

[9] H. Liu and J. L. Richards, "Hydraulics of stationary capsule in pipe," Journal of Hydraulic Engineering, vol. 120, no. 1, pp. 22-40, 1994.

[10] H. Liu and H. R. Graze, "Lift and drag on stationary capsule in pipeline," Journal of Hydraulic Engineering, vol. 109, no. 1, pp. 28-47, 1983.

[11] Y. Tomita, M. Yamamoto, and K. Funatsu, "Motion of a single capsule in a hydraulic pipeline," Journal of Fluid Mechanics, vol. 171, no. -1, pp. 495-508, 1986.

[12] Y. Tomita, T. Okubo, and K. Funatsu, "Unsteady analysis of hydraulic capsule transport in a straight horizontal pipeline," in Proceedings of the 6th International Symposium on Freight Pipelines, pp. 273-278, Columbia, MO, USA, May 1989.

[13] P. Vlasak, "An experimental investigation of capsules of anomalous shape conveyed by liquid in a pipe," Powder Technology, vol. 104, no. 3, pp. 207-213, 1999.

[14] B. Latto and K. W. Chow, "Hydrodynamic transport of cylindrical capsules in a vertical pipeline," The Canadian Journal of Chemical Engineering, vol. 60, no. 6, pp. 713-722, 1982.

[15] D. Ulusarslan and İ. Teke, "An experimental investigation of the capsule velocity, concentration rate and the spacing between the capsules for spherical capsule train flow in a horizontal circular pipe," Powder Technology, vol. 159, no. 1, pp. 27-34, 2005.

[16] T. Asim and R. Mishra, "Development of a design methodology for hydraulic pipelines carrying rectangular capsules," International Journal of Pressure Vessels and Piping, vol. 146, pp. 111-128, 2006.

[17] D. Ulusarslan, "Analysis of the pressure gradients according to capsule flow mechanism," Particle \& Particle Systems Characterization, vol. 29, no. 3, pp. 167-177, 2012.

[18] M. C. Robert and R. Nivedita, "Gupta Inertial effects on the flow of capsules in cylindrical channels," International Journal of Multiphase Flow, vol. 87, pp. 114-123, 2016.

[19] F. Mohamed, S. Z. Khalil, and I. G. Kassab, “Turbulent flow around single concentric long capsule in a pipe," Applied Mathematical Modelling, vol. 34, no. 8, pp. 2000-2017, 2010.
[20] T. Asim, Computational fluid dynamics based diagnostics and optimal design of hydraulic capsule pipelines, Ph.D. thesis, University of Huddersfield, Huddersfield, UK, 2013.

[21] T. Asim, A. Algadi, and R. Mishra, "Effect of capsule shape on hydrodynamic characteristics and optimal design of hydraulic capsule pipelines," Journal of Petroleum Science and Engineering, vol. 161, pp. 390-408, 2018.

[22] T. Asim and R. Mishra, "Optimal design of hydraulic capsule pipelines transporting spherical capsules," The Canadian Journal of Chemical Engineering, vol. 94, no. 5, pp. 966-979, 2016.

[23] P. F. Zhang, J. J. Wang, and L. X. Huang, "Numerical simulation of flow around cylinder with an upstream rod in tandem at low Reynolds numbers," Applied Ocean Research, vol. 28, no. 3, pp. 183-192, 2006.

[24] L. Zhang, C. N. Ji, and G. Y. Xing, "Investigation on near-wake patterns and hydrodynamic coefficients of flow around three side-by-side cylinders with a low Reynolds number," Shuidonglixue Yanjiu Yu Jinzhan/Chinese Journal of Hydrodynamics, vol. 32, no. 3, pp. 263-272, 2017.

[25] T. Vadim, K. Eckert, and S. Odenbach, "Linear stability analysis of the convective flow in a spherical gap with $\eta=714$," International Journal of Heat and Mass Transfer, vol. 80, pp. 266-273, 2015.

[26] Y. H. Jing, X. H. Guo, X. H. Sun et al., "Hydraulic characteristics of cyclical slit flow for piped carriage," Water Resources and Power, vol. 32, no. 7, pp. 151-155, 2014.

[27] A. P. Polikarpov and I. Graur, "Unsteady rarefied gas flow through a slitfied gas flow through a slit," Vacuum, vol. 101, pp. 79-85, 2014.

[28] E. S. Winkel, J. M. Cutbirth, S. L. Ceccio, M. Perlin, and D. R. Dowling, "Turbulence profiles from a smooth flat-plate turbulent boundary layer at high Reynolds number," Experimental Thermal and Fluid Science, vol. 40, pp. 140-149, 2012.

[29] S. Philipp and R. Orlu, "Assessment of direct numerical simulation data of turbulent boundary layers," Journal of Fluid Mechanics, vol. 659, pp. 116-126, 2010.

[30] C. J. Zhang, X. H. Sun, Y. Y. Li et al., "Effect of fluid-structure interaction on internal flow field characteristics of tubecontained raw material pipeline hydraulic transportation," Transactions of the Chinese Society of Agricultural Engineering, vol. 34, no. 18, pp. 299-307, 2018.

[31] H. Tao, "Numerical solution techniques for fluid-structure interaction simulations: a brief review and discussion," Journal of Vibration and Shock, vol. 37, no. 4, pp. 184-190, 2018.

[32] X. H. Sun, Y. Y. Li, and Q. F. Yan, "Experimental study on starting conditions of the hydraulic transportation on the piped carriage," in Proceedings of the 20th National Conference on Hydrodynamics, pp. 425-431, Taiyuan, China, 2007.

[33] Y. Y. Li, X. H. Sun, and Y. F. Yan, "Hydraulic characteristics of tube-contained raw material hydraulic transportation under different loads on the piped carriage," Transactions of the Chinese Society of Agricultural Engineering, vol. 39, pp. 93-96, 2008.

[34] X. N. Yang, "Study on the character of spiral flow caused by different length guide vanes," Master's Thesis, Taiyuan University of Technology, Taiyuan, China, 2013.

[35] Y. Y. Li and X. H. Sun, "Hydraulic characteristics of transportation of different piped carriages in pipe," Journal of Drainage and Irrigation Machinery Engineering, vol. 28, pp. 174-178, 2010.

[36] R. Wang, X. H. Sun, and Y. Y. Li, "Transportation characteristics of piped carriage with different Reynolds numbers," 
Journal of Drainage and Irrigation Machinery Engineering, vol. 29, pp. 343-346, 2011.

[37] X.-l. Zhang, X.-H. Sun, Y.-Y. Li, X.-N. Xi, F. Guo, and L.-J. Zheng, "Numerical investigation of the concentric annulus flow around a cylindrical body with contrasted effecting factors," Journal of Hydrodynamics, vol. 27, no. 2, pp. 273285, 2015.

[38] X.-L. Zhang, X.-H. Sun, and Y.-Y. Li, "3-D numerical investigation of the wall-bounded concentric annulus flow around a cylindrical body with a special array of cylinders," Journal of Hydrodynamics, vol. 27, no. 1, pp. 120-130, 2015.

[39] T. Asim and R. Mishra, "Computational fluid dynamics based optimal design of hydraulic capsule pipelines transporting cylindrical capsules," Powder Technology, vol. 295, pp. 180201, 2016.

[40] D. Ulusarslan and I. Teke, "An experimental determination of pressure drops in the flow of low density spherical capsule train inside horizontal pipes," Experimental Thermal and Fluid Science, vol. 30, no. 3, pp. 233-241, 2006.

[41] D. D. Joseph, R. Bai, K. P. Chen, and Y. Y. Renardy, "Coreannular flows," Annual Review of Fluid Mechanics, vol. 29, no. 1, pp. 65-90, 1997.

[42] K. C. Sahu, "Linear instability in a miscible core-annular flow of a Newtonian and a Bingham fluid," Journal of Non-Newtonian Fluid Mechanics, vol. 264, pp. 159-169, 2019.

[43] R. Usha and K. C. Sahu, "Interfacial instability in pressuredriven core-annular pipe flow of a Newtonian and a HerschelBulkley fluid," Journal of Non-Newt Fluid Mechanics, vol. 271, pp. 104-144, 2019.

[44] J. Z. Lin, X. D. Ruan, B. G. Chen, J. P. Wang, J. Zhou, and A. L. Ren, Fluid Mechanics, Tsinghua University Press, Beijing, China, 2nd edition, 2005.

[45] Z. C. Zhang, G. D. Li, and Z. Q. Li, Hydraulics, Vol. 1, China Water \& Power Press, Beijing, China, 2011. 